\title{
Palladium(II)-catalyzed Cross-Dehydrogenative Coupling (CDC) of $N$-Phthaloyl Dehydroalanine Esters with Simple Arenes: Stereoselective Synthesis of Z- Dehydrophenylalanine Derivatives
}

\author{
Francesca Bartoccini, Diego Maria Cannas, Francesco Fini, Giovanni Piersanti* \\ Department of Biomolecular Sciences \\ University of Urbino "Carlo Bo" \\ P.zza Rinascimento 6, 61029 Urbino (PU), Italy \\ *e-mail: giovanni.piersanti@uniurb.it
}

Table of Contents

1. General Methods $\quad$ S2

2. Starting Materials $\quad$ S2

3. Synthesis and characterization data of compounds 1d $\quad$ S2

$\begin{array}{ll}\text { 4. General Procedure for small scale } & \text { S3 }\end{array}$

5. Characterization data of compounds 3a, 4a and 5a-n $\quad$ S3

6. Procedure for large scale synthesis of compound $5 f \quad S 11$

7. Synthesis and Characterization data of compounds 7-10 $\quad$ S11

8. References $\quad$ S14

9. Copies of ${ }^{1}$ H NMR and ${ }^{13}$ C NMR spectra $\quad$ S15 
General Methods. All reactions were run in air unless otherwise noted. Column chromatography purifications were performed in flash chromatography conditions using Merck 230-400 Mesh silica gel. Analytical thin layer chromatography (TLC) was carried out on Merck silica gel plates (Silica Gel 60 F254), that were visualized by exposure to ultraviolet light and an aqueous solution of $\mathrm{KMnO}_{4} \cdot{ }^{1} \mathrm{H} \mathrm{NMR},{ }^{13} \mathrm{C} \mathrm{NMR}$ and ${ }^{19} \mathrm{~F}$ NMR spectra were recorded on a Bruker Avance 400 spectrometer, using $\mathrm{CDCl}_{3}$ and $\mathrm{CD}_{3} \mathrm{OD}$ as solvent. Chemical shifts ( $\delta$ scale) are reported in parts per million (ppm) relative to the central peak of the solvent. Coupling constants ( $J$ values) are given in hertz (Hz). ESI-MS spectra was taken on a Waters Micromass ZQ instrument. IR spectra were obtained on a Nicolet Avatar 360 FT-IR spectrometer, absorbance are reported in $\mathrm{cm}^{-1}$. Melting points were determined on a Buchi SMP-510 capillary melting point apparatus and are uncorrected. Elemental analyses were performed on a Carlo Erba analyzer, and the results are within \pm 0.4 of the theoretical values $(\mathrm{C}, \mathrm{H}, \mathrm{N})$.

Starting Materials. Methyl 2-acetamidoacrylate (1a), arene derivatives, bis(pinacolato)diboron $\left(\mathrm{B}_{2} \mathrm{pin}_{2}\right)$, (dimethylphenylsilyl)boronic acid pinacol ester $\left(\mathrm{Me}_{2} \mathrm{PhSi}-\mathrm{Bpin}\right)$ and diethoxymethylsilane (DEMS) are commercially available. Methyl 2-(4-methylphenylsulfonamido)acrylate (1b), ${ }^{1}$ methyl 2-(((9H-fluoren-9-yl)methoxy)carbonylamino)acrylate ${ }^{2} \quad$ (1c), methyl 2-(2-oxooxazolidin-3yl)acrylate ${ }^{3}(\mathbf{1 e})$ and methyl 2-(1,3-dioxoisoindolin-2-yl)acrylate ${ }^{4}$ (1f) were prepared as reported in the literature.

\section{Methyl 2-(2-oxopyrrolidin-1-yl)acrylate (1d) ${ }^{5}$}

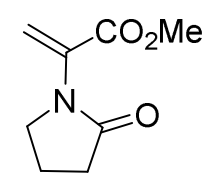

A round-bottom flask equipped with a Dean-Stark trap was charged successively with pyrrolidin-2one (232 mg, $2.7 \mathrm{mmol})$, methyl pyruvate $(0.2 \mathrm{~mL}, 3 \mathrm{mmol})$, a catalytic amount of p-TsOH (27 mg, $0.14 \mathrm{mmol})$ and toluene $(9 \mathrm{~mL})$. The stirred mixture was heated under reflux for $24 \mathrm{~h}$ then concentrated in vacuo. The residue obtained was purified by flash chromatography (cyclohexane/ ethyl acetate $1: 1)$ to give 1 e $(207 \mathrm{mg}, 45 \%)$ as colourless oil.

TLC (cyclohexane/ ethyl acetate 1:1): $R_{\mathrm{f}}=0.25\left(\mathrm{UV}, \mathrm{KMnO}_{4}\right) ;{ }^{1} \mathrm{H} \mathrm{NMR}\left(400 \mathrm{MHz}, \mathrm{CDCl}_{3}\right): \delta 5.89$ $(\mathrm{d}, J=0.5 \mathrm{~Hz}, 1 \mathrm{H}), 5.44(\mathrm{~d}, J=0.5 \mathrm{~Hz}, 1 \mathrm{H}), 3.77(\mathrm{~s}, 3 \mathrm{H}), 3.61(\mathrm{t}, J=7.5 \mathrm{~Hz}, 2 \mathrm{H}), 2.45(\mathrm{t}, J=7.5$ $\mathrm{Hz}, 2 \mathrm{H}), 2.15-2.07$ (m, 2H); ${ }^{13} \mathrm{C} \mathrm{NMR}\left(100 \mathrm{MHz}, \mathrm{CDCl}_{3}\right): \delta 174.5,164.1,135.9,116.4,52.4$, 48.5, 30.9, 18.4; IR (film): 1723, $1690 \mathrm{~cm}^{-1}$; MS (ESI): $\mathrm{m} / \mathrm{z}=170$ [M + 1]; $\mathrm{C}_{8} \mathrm{H}_{11} \mathrm{NO}_{3}$ (169.07): calcd. C, 56.80; H, 6.55; N, 8.28; found C, 56.67; H, 6.49; N, 8.33. 
General Procedure for small scale. Methyl 2-(1,3-dioxoisoindolin-2-yl)acrylate (1f) (69 mg, 0.3 $\mathrm{mmol})$, arene derivatives, $\mathrm{Pd}(\mathrm{OAc})_{2}(3.4 \mathrm{mg}, 0.015 \mathrm{mmol})$, 3,5-dichloropyridine $(2.2 \mathrm{mg}, 0.015$ mmol), tert-butyl peroxybenzoate $(57 \mu \mathrm{L}, 0.3 \mathrm{mmol})$, and acetic acid $(1.4 \mathrm{~mL})$ were combined in a $5 \mathrm{~mL}$ vial equipped with a magnetic stir bar. The reaction was stirred at $100{ }^{\circ} \mathrm{C}$ for $16 \mathrm{~h}$. The resulting reaction mixture was diluted with ethyl acetate $(5 \mathrm{~mL})$ and filtered under a plug of silica gel. The solvents are concentrated under reduced pressure. The residue obtained was purified by flash chromatography.

Methyl (Z)-2-(2-oxopyrrolidin-1-yl)-3-phenylacrylate (3a)<smiles>COC(=O)/C=C(/c1ccccc1)N1CCCC1=O</smiles>

The title compound was prepared according to the general procedure using methyl 2-(2oxopyrrolidin-1-yl)acrylate (1e) and benzene (2a) $(0.3 \mathrm{~mL}, 3.38 \mathrm{mmol})$. The product was purified by flash chromatography (cyclohexane/ ethyl acetate 1:1) to give $\mathbf{3 a}(48 \mathrm{mg}, 66 \%)$ as colourless oil. TLC (cyclohexane/ ethyl acetate 1:1): $R_{\mathrm{f}}=0.35\left(\mathrm{UV}, \mathrm{KMnO}_{4}\right) ;{ }^{1} \mathrm{H} \mathrm{NMR}\left(400 \mathrm{MHz}, \mathrm{CDCl}_{3}\right): \delta 7.69$ (s, 1H), $7.51-7.48(\mathrm{~m}, 2 \mathrm{H}), 7.39-7.37(\mathrm{~m}, 3 \mathrm{H}), 3.84(\mathrm{~s}, 3 \mathrm{H}), 3.49(\mathrm{t}, J=7.5 \mathrm{~Hz}, 2 \mathrm{H}), 2.52(\mathrm{t}, J=$ $7.5 \mathrm{~Hz}, 2 \mathrm{H}), 2.20-2.12(\mathrm{~m}, 2 \mathrm{H}) ;{ }^{13} \mathrm{C} \mathrm{NMR}\left(100 \mathrm{MHz}, \mathrm{CDCl}_{3}\right): \delta 176.4,164.6,138.7,132.9$, 130.2, 129.5, 128.8, 126.9, 52.6, 47.9, 30.7, 19.3; IR (film): 1728, $1695 \mathrm{~cm}^{-1}$; MS (ESI): m/z = 246 $[\mathrm{M}+1] ; \mathrm{C}_{14} \mathrm{H}_{15} \mathrm{NO}_{3}(245.11)$ : calcd. C, 68.56; H, 6.16; N, 5.71; found C, 68.65; H, 6.20; N, 5.66.

Methyl (Z)-2-(2-oxooxazolidin-3-yl)-3-phenylacrylate (4a)<smiles>COC(=O)/C(=C/c1ccccc1)N1CCOC1=O</smiles>

The title compound was prepared according to the general procedure using methyl 2-(2oxooxazolidin-3-yl)acrylate (1f) and benzene (2a) $(0.3 \mathrm{~mL}, 3.38 \mathrm{mmol})$. The product was purified by flash chromatography (cyclohexane/ ethyl acetate 4:6) to give $\mathbf{4 a}(20 \mathrm{mg}, 35 \%)$ as colourless oil. TLC (cyclohexane/ ethyl acetate 4:6): $R_{\mathrm{f}}=0.5\left(\mathrm{UV}, \mathrm{KMnO}_{4}\right) ;{ }^{1} \mathrm{H} \mathrm{NMR}\left(400 \mathrm{MHz}, \mathrm{CDCl}_{3}\right): \delta 7.72$ (s, 1H), $7.60-7.57(\mathrm{~m}, 2 \mathrm{H}), 7.43-7.41(\mathrm{~m}, 3 \mathrm{H}), 4.50(\mathrm{t}, J=8.0 \mathrm{~Hz}, 2 \mathrm{H}), 3.88(\mathrm{~s}, 3 \mathrm{H}), 3.72(\mathrm{t}, J=$ $8.0 \mathrm{~Hz}, 2 \mathrm{H}) ;{ }^{13} \mathrm{C}$ NMR $\left(100 \mathrm{MHz}, \mathrm{CDCl}_{3}\right): \delta 164.4,157.5,139.9,132.4,130.6,129.9,129.0,125.4$, 63.0, 52.7, 45.4; IR (film): 1754, $1711 \mathrm{~cm}^{-1}$; MS (ESI): $\mathrm{m} / \mathrm{z}=248[\mathrm{M}+1] ; \mathrm{C}_{13} \mathrm{H}_{13} \mathrm{NO}_{4}$ (247.08): calcd. C, 63.15; H, 5.30; N, 5.67; found C, 63.29; H, 5.36; N, 5.62.

\section{Methyl (Z)-2-(1,3-dioxoisoindolin-2-yl)-3-phenylacrylate (5a)}


If $\mathrm{CO}_{2} \mathrm{Me}$

The title compound was prepared according to the general procedure using benzene (2a) $(0.3 \mathrm{~mL}$, $3.38 \mathrm{mmol}$ ). The product was purified by flash chromatography (cyclohexane/ ethyl acetate 9:1) to give $5 \mathbf{a}(87 \mathrm{mg}, 95 \%)$ as white solid.

TLC (cyclohexane/ ethyl acetate 9:1): $R_{\mathrm{f}}=0.14\left(\mathrm{UV}, \mathrm{KMnO}_{4}\right) ;{ }^{1} \mathrm{H}$ NMR $\left(400 \mathrm{MHz}, \mathrm{CDCl}_{3}\right): \delta$ $8.13(\mathrm{~s}, 1 \mathrm{H}), 7.93(\mathrm{dd}, J=5.5,3.0 \mathrm{~Hz}, 2 \mathrm{H}), 7.80(\mathrm{dd}, J=5.5,3.0 \mathrm{~Hz}, 2 \mathrm{H}), 7.42-7.40(\mathrm{~m}, 2 \mathrm{H}), 7.34$ $-7.30(\mathrm{~m}, 3 \mathrm{H}), 3.84(\mathrm{~s}, 3 \mathrm{H}) ;{ }^{13} \mathrm{C} \mathrm{NMR}\left(100 \mathrm{MHz} \mathrm{CDCl}_{3}\right): \delta 166.8,163.9,143.1,134.5,132.3$, 132.1, 130.6, 129.4, 128.9, 124.0, 120.0, 52.9; m.p. 123-125 ${ }^{\circ} \mathrm{C}$; IR (film): 1761, $1727 \mathrm{~cm}^{-1}$; MS (ESI): $\mathrm{m} / \mathrm{z}=308[\mathrm{M}+1] ; \mathrm{C}_{18} \mathrm{H}_{13} \mathrm{NO}_{4}$ (307.08): calcd. C, 70.35; H, 4.26; N, 4.56; found $\mathrm{C}, 70.51$; H, 4.18; N, 4.51.

\section{Methyl (Z)-2-(1,3-dioxoisoindolin-2-yl)-3-(o-/m-/p-tolyl)acrylate (5b)}<smiles>CC(=O)c1ccccc1NC(=O)c1ccc(C)cc1</smiles>

The title compound was prepared according to the general procedure using toluene $(0.3 \mathrm{~mL}, 2.8$ mmol). The product was purified by flash chromatography (cyclohexane/ ethyl acetate 8:2) to give $\mathbf{5 b}(83 \mathrm{mg}$, as a mixture of product isomers, $86 \%$ yield, 1:1.2:1.3 $\mathrm{o}: \mathrm{m}: \mathrm{p})$ as white solid.

TLC (cyclohexane/ ethyl acetate 8:2): $R_{\mathrm{f}}=0.36\left(\mathrm{UV}, \mathrm{KMnO}_{4}\right) ;{ }^{1} \mathrm{H}$ NMR $\left(400 \mathrm{MHz}, \mathrm{CDCl}_{3}\right): \delta 8.27$ $(\mathrm{s}, 0.8 \mathrm{H}), 8.095(\mathrm{~s}, 0.9 \mathrm{H}), 8.089(\mathrm{~s}, 1 \mathrm{H}), 7.93-7.91(\mathrm{~m}, 3.8 \mathrm{H}), 7.83(\mathrm{dd}, J=5.5,3.0 \mathrm{~Hz}, 1.6 \mathrm{H})$, $7.80-7.78(\mathrm{~m}, 3.8 \mathrm{H}), 7.72(\mathrm{dd}, J=5.5,3.0 \mathrm{~Hz}, 1.6 \mathrm{H}), 7.30(\mathrm{~d}, J=8.0 \mathrm{~Hz}, 2 \mathrm{H}), 7.21-7.14(\mathrm{~m}$, 6H), 7.09 (d, $J=8.0 \mathrm{~Hz}, 2 \mathrm{H}), 7.01-6.96(\mathrm{~m}, 0.8 \mathrm{H}), 3.85(\mathrm{~s}, 2.4 \mathrm{H}), 3.82(\mathrm{~s}, 3 \mathrm{H}), 3.82(\mathrm{~s}, 2.7 \mathrm{H})$, $2.43(\mathrm{~s}, 2.4 \mathrm{H}), 2.29(\mathrm{~s}, 2.7 \mathrm{H}), 2.24(\mathrm{~s}, 3 \mathrm{H}) ;{ }^{13} \mathrm{C} \mathrm{NMR}\left(100 \mathrm{MHz}, \mathrm{CDCl}_{3}\right): \delta 166.9,166.8,166.7$, $164.1,164.0,163.7,143.3,143.1,143.0,141.3,138.5,137.6,134.50,134.49,134.3,132.24$, $132.17,132.2$, 132.1, 132.0, 131.5, 130.6, 130.4, 129.8, 129.7, 129.6, 129.5, 128.8, 126.8, 126.01, $125.98,124.00,123.99,123.8,121.5,119.8,119.0,52.89,52.87,52.8,21.5,21.3,20.0$; IR (film): 1783, $1721 \mathrm{~cm}^{-1}$; MS (ESI): m/z =322 [M + 1], 290 [M - 31]; $\mathrm{C}_{19} \mathrm{H}_{15} \mathrm{NO}_{4}$ (321.10): calcd. C, 71.02; $\mathrm{H}, 4.71 ; \mathrm{N}, 4.36$; found $\mathrm{C}, 70.82 ; \mathrm{H}, 4.62 ; \mathrm{N}, 4.43$.

Methyl (Z)-2-(1,3-dioxoisoindolin-2-yl)-3-(2-/3-/4-methoxyphenyl)acrylate (5c)<smiles>COc1ccc(C(=O)Nc2ccccc2)cc1</smiles> 
The title compound was prepared according to the general procedure using anisole $(0.3 \mathrm{~mL}, 2.76$ mmol). The product was purified by flash chromatography (cyclohexane/ ethyl acetate 8:2) to give 5c (88 $\mathrm{mg}$, as a mixture of product isomers, 87\% yield, 1.7:1:2.7 o:m:p) as white solid.

TLC (cyclohexane/ ethyl acetate 8:2): $R_{\mathrm{f}}=0.18\left(\mathrm{UV}, \mathrm{KMnO}_{4}\right) ;{ }^{1} \mathrm{H} \mathrm{NMR}\left(400 \mathrm{MHz}, \mathrm{CDCl}_{3}\right): \delta 8.39$ $(\mathrm{s}, 0.6 \mathrm{H}), 8.10(\mathrm{~s}, 0.4 \mathrm{H}), 8.10-8.08(\mathrm{~m}, 0.4 \mathrm{H}), 8.05(\mathrm{~s}, 1 \mathrm{H}), 7.93-7.89(\mathrm{~m}, 2.8 \mathrm{H}), 7.87(\mathrm{dd}, J=$ $5.5,3.0 \mathrm{~Hz}, 1.2 \mathrm{H}), 7.81-7.76(\mathrm{~m}, 2.8 \mathrm{H}), 7.74(\mathrm{dd}, J=5.5,3.0 \mathrm{~Hz}, 1.2 \mathrm{H}), 7.61-7.57(\mathrm{~m}, 0.4 \mathrm{H})$, $7.47-7.44(\mathrm{~m}, 0.8 \mathrm{H}), 7.38(\mathrm{~d}, J=8.0 \mathrm{~Hz}, 2 \mathrm{H}), 7.26-7.24(\mathrm{~m}, 1.2 \mathrm{H}), 6.87-6.84(\mathrm{~m}, 0.6 \mathrm{H}), 6.79$ $(\mathrm{d}, J=8.0 \mathrm{~Hz}, 2 \mathrm{H}), 6.74(\mathrm{t}, J=8.0 \mathrm{~Hz}, 0.6 \mathrm{H}), 3.82-3.80(\mathrm{~m}, 8 \mathrm{H}), 3.74(\mathrm{~s}, 3 \mathrm{H}), 3.61(\mathrm{~s}, 1.2 \mathrm{H})$;

${ }^{13} \mathrm{C}$ NMR $\left(100 \mathrm{MHz}, \mathrm{CDCl}_{3}\right): \delta 171.1,167.0,166.8,164.3,164.0,163.9,161.6,159.6,158.0$, $143.0,142.6,138.6,134.6,134.5,134.4,133.7,132.2,132.1,132.0,131.7,130.1,130.0,129.4$, $128.5,128.4,124.7,124.0,123.8,121.7,121.6,120.7,120.2,119.9,117.4,116.6,114.5,114.3$, 111.1, 55.7, 55.3, 55.0, 52.9, 52.8, 52.7; IR (film): 1786, $1721 \mathrm{~cm}^{-1} ; \mathrm{MS}$ (ESI): m/z=338 [M + 1], 306 [M - 31]; $\mathrm{C}_{19} \mathrm{H}_{15} \mathrm{NO}_{5}$ (337.10): calcd. C, 67.65; H, 4.48; N, 4.15; found $\mathrm{C}, 67.48$.; H, 4.38; N, 4.09 .

\section{Methyl (Z)-3-(2,5-dimethylphenyl)-2-(1,3-dioxoisoindolin-2-yl)acrylate (5d)}<smiles>CC(=O)C(=Cc1cc(C)ccc1C)[NH+]([O-])c1ccccc1</smiles>

The title compound was prepared according to the general procedure using p-xylene $(0.3 \mathrm{~mL}, 2.43$ mmol). The product was purified by flash chromatography (gradient from cyclohexane/ ethyl acetate 9:1 to cyclohexane/ ethyl acetate 8:2) to give $\mathbf{5 d}(80 \mathrm{mg}, 80 \%)$ as white solid.

TLC (cyclohexane/ ethyl acetate 9:1): $R_{\mathrm{f}}=0.2\left(\mathrm{UV}, \mathrm{KMnO}_{4}\right) ;{ }^{1} \mathrm{H} \mathrm{NMR}\left(400 \mathrm{MHz}, \mathrm{CDCl}_{3}\right): \delta 8.25$ (s, 1H), $7.86(\mathrm{dd}, J=5.5,3.0 \mathrm{~Hz}, 2 \mathrm{H}), 7.74(\mathrm{dd}, J=5.5,3.0 \mathrm{~Hz}, 2 \mathrm{H}), 7.08(\mathrm{~d}, J=7.5 \mathrm{~Hz}, 1 \mathrm{H}), 6.98$ $(\mathrm{d}, J=7.5 \mathrm{~Hz}, 1 \mathrm{H}), 6.94(\mathrm{~s}, 1 \mathrm{H}), 3.85(\mathrm{~s}, 3 \mathrm{H}), 2.38(\mathrm{~s}, 3 \mathrm{H}), 2.06(\mathrm{~s}, 3 \mathrm{H}) ;{ }^{13} \mathrm{C} \mathrm{NMR}(100 \mathrm{MHz}$, $\left.\mathrm{CDCl}_{3}\right): \delta 166.8,163.8,143.2,135.3,134.5,134.3,132.01,131.97,130.6,130.2,127.4,123.8$, 121.3, 52.8, 20.8, 19.5; m.p. 132-134 ${ }^{\circ} \mathrm{C}$; IR (film): 1785, $1720 \mathrm{~cm}^{-1}$; MS (ESI): $\mathrm{m} / \mathrm{z}=336[\mathrm{M}+$ 1], 304 [M - 31]; $\mathrm{C}_{20} \mathrm{H}_{17} \mathrm{NO}_{4}$ (335.12): calcd. C, 71.63; H, 5.11; N, 4.18; found C, 71.86; H, 5.18; $\mathrm{N}, 4.10$.

\section{Methyl (Z)-3-(2,5-dimethoxyphenyl)-2-(1,3-dioxoisoindolin-2-yl)acrylate (5e)}<smiles>COc1ccc(OC)c(C=C(C(C)=O)[NH+](c2ccccc2)c2ccccc2)c1</smiles> 
The title compound was prepared according to the general procedure using 1,4-dimethoxybenzene $(0.3 \mathrm{~mL}, 2.29 \mathrm{mmol})$. The product was purified by flash chromatography (cyclohexane/ ethyl acetate $7: 3)$ to give $\mathbf{5 e}(81 \mathrm{mg}, 74 \%)$ as yellow solid.

TLC (cyclohexane/ ethyl acetate 7:3): $R_{\mathrm{f}}=0.3\left(\mathrm{UV}, \mathrm{KMnO}_{4}\right) ;{ }^{1} \mathrm{H} \mathrm{NMR}\left(400 \mathrm{MHz}, \mathrm{CDCl}_{3}\right): \delta 8.36$ (s, 1H), $7.89(\mathrm{dd}, J=5.5,3.0 \mathrm{~Hz}, 2 \mathrm{H}), 7.89(\mathrm{dd}, J=5.5,3.0 \mathrm{~Hz}, 2 \mathrm{H}), 6.87-6.80(\mathrm{~m}, 3 \mathrm{H}), 3.84$ (s, 4H), 3.79 (s, 3H), $3.46(\mathrm{~s}, 3 \mathrm{H}) ;{ }^{13} \mathrm{C}$ NMR (100 MHz, $\left.\mathrm{CDCl}_{3}\right): \delta 166.7,164.0,153.2,152.5,138.5$, 134.4, 132.1, 123.8, 122.1, 120.2, 118.0, 112.9, 112.4, 56.3, 55.4, 52.8; m.p. 188-190 ${ }^{\circ} \mathrm{C}$; IR (film): 1785, $1721 \mathrm{~cm}^{-1} ; \mathrm{MS}(\mathrm{ESI}): \mathrm{m} / \mathrm{z}=368[\mathrm{M}+1], 336[\mathrm{M}-31] ; \mathrm{C}_{20} \mathrm{H}_{17} \mathrm{NO}_{6}$ (367.11): calcd. C, 65.39; H, 4.66; N, 3.81; found C, 65.57; H, 4.75; N, 3.74.

\section{Methyl (Z)-2-(1,3-dioxoisoindolin-2-yl)-3-(2,4,6-trifluorophenyl)acrylate (5f)}<smiles>CC(=O)/C(=C/c1c(F)cc(F)cc1F)[PH+]c1ccccc1</smiles>

The title compound was prepared according to the general procedure using 1,3,5-trifluorobenzene $(0.3 \mathrm{~mL}, 2.9 \mathrm{mmol})$. The product was purified by flash chromatography (gradient from cyclohexane/ ethyl acetate 9:1 to cyclohexane/ ethyl acetate 8:2) to give $\mathbf{5 f}(61 \mathrm{mg}, 56 \%)$ as white solid.

TLC (cyclohexane/ ethyl acetate 8:2): $R_{\mathrm{f}}=0.29\left(\mathrm{UV}, \mathrm{KMnO}_{4}\right) ;{ }^{1} \mathrm{H}$ NMR $\left(400 \mathrm{MHz}, \mathrm{CDCl}_{3}\right): \delta 7.86$ $(\mathrm{dd}, J=5.5,3.0 \mathrm{~Hz}, 2 \mathrm{H}), 7.83(\mathrm{~s}, 1 \mathrm{H}), 7.76(\mathrm{dd}, J=5.5,3.0 \mathrm{~Hz}, 2 \mathrm{H}), 6.64(\mathrm{t}, J=8.5 \mathrm{~Hz}, 2 \mathrm{H}), 3.86$ $(\mathrm{s}, 3 \mathrm{H}) ;{ }^{13} \mathrm{C}$ NMR $\left(100 \mathrm{MHz}, \mathrm{CDCl}_{3}\right): \delta 165.5,163.6(\mathrm{dt}, J=251,16 \mathrm{~Hz}), 163.0,160.6(\mathrm{ddd}, J=$ 253, 14, $9 \mathrm{~Hz}), 134.5,131.8,127.6,124.8,123.9,107.8$ (td, $J=18,4 \mathrm{~Hz}), 100.9$ (td, $J=26,4 \mathrm{~Hz})$, 53.1; ${ }^{19} \mathrm{~F}$ NMR (377 MHz, $\mathrm{CDCl}_{3}$ ): $\delta-104.0$ (quin, $\left.J=8.5 \mathrm{~Hz}, 1 \mathrm{~F}\right),-104.6$ (t, $J=8.5 \mathrm{~Hz}, 2 \mathrm{~F}$ ); m.p. 156-158 ${ }^{\circ} \mathrm{C}$; IR (film): 1792, $1724 \mathrm{~cm}^{-1}$; MS (ESI): m/z = $362[\mathrm{M}+1], 330[\mathrm{M}-31] ; \mathrm{C}_{18} \mathrm{H}_{10} \mathrm{~F}_{3} \mathrm{NO}_{4}$ (361.06): calcd. C, 59.84; H, 2.79; N, 3.88; found C, 59.63; H, 2.83; N, 3.96.

Methyl (Z)-2-(1,3-dioxoisoindolin-2-yl)-3-(perfluorophenyl)acrylate (5g)<smiles>COC(=O)/C(=C/c1c(F)c(F)c(F)c(F)c1F)[NH+](c1ccccc1)c1ccccc1</smiles>

The title compound was prepared according to the general procedure using 1,2,3,4,5pentafluorobenzene $(1.1 \mathrm{~mL}, 9.9 \mathrm{mmol})$. The product was purified by flash chromatography 
(gradient from cyclohexane/ ethyl acetate 9:1 to cyclohexane/ ethyl acetate 8:2) to give $\mathbf{5 g}$ (55 $\mathrm{mg}$, $46 \%$ ) as white solid.

TLC (cyclohexane/ ethyl acetate 8:2): $R_{\mathrm{f}}=0.36\left(\mathrm{UV}, \mathrm{KMnO}_{4}\right) ;{ }^{1} \mathrm{H}$ NMR $\left(400 \mathrm{MHz}, \mathrm{CDCl}_{3}\right): \delta 7.89$ $(\mathrm{dd}, J=5.5,3.0 \mathrm{~Hz}, 2 \mathrm{H}), 7.80(\mathrm{dd}, J=5.5,3.0 \mathrm{~Hz}, 2 \mathrm{H}), 7.74(\mathrm{~s}, 1 \mathrm{H}), 3.89(\mathrm{~s}, 3 \mathrm{H}) ;{ }^{13} \mathrm{C} \mathrm{NMR}(100$ $\left.\mathrm{MHz}, \mathrm{CDCl}_{3}\right): \delta 165.3,162.5,144.4(\mathrm{dm}, J=250 \mathrm{~Hz}), 141.9(\mathrm{dm}, J=244 \mathrm{~Hz}), 137.8(\mathrm{dm}, J=235$ $\mathrm{Hz}), 134.7,131.7,126.9,124.9,124.1,108.9$ (dt, $J=16.5,4 \mathrm{~Hz}), 53.4 ;{ }^{19} \mathrm{~F}$ NMR (377 MHz, $\left.\mathrm{CDCl}_{3}\right): \delta-136.6(\mathrm{~d}, J=17.5 \mathrm{~Hz}, 2 \mathrm{~F}),-150.8(\mathrm{t}, J=20.5 \mathrm{~Hz}, 1 \mathrm{~F}),-160.9(\mathrm{t}, J=20.5 \mathrm{~Hz}, 2 \mathrm{~F}) ;$ m.p. 192-194 ${ }^{\circ} \mathrm{C}$; IR (film): 1784, $1721 \mathrm{~cm}^{-1}$; MS (ESI): m/z = $398[\mathrm{M}+1] ; \mathrm{C}_{18} \mathrm{H}_{8} \mathrm{~F}_{5} \mathrm{NO}_{4}$ (397.04): calcd. C, 54.42; H, 2.03; N, 3.53; found C, 54.25; H, 2.06; N, 3.58 .

Methyl (Z)-2-(1,3-dioxoisoindolin-2-yl)-3-(naphthalen-1-/2-yl)acrylate (5h)<smiles>CC(=O)C(=Cc1ccccc1)Nc1ccccc1</smiles>

The title compound was prepared according to the general procedure using naphthalene $(384 \mathrm{mg}, 3$ mmol). The product was purified by flash chromatography (cyclohexane/ ethyl acetate 8:2) to give 5h $(89 \mathrm{mg}$, as a mixture of product isomers, $83 \%$ yield, 1.4:1 $\alpha: \beta)$ as off-white solid.

TLC (cyclohexane/ ethyl acetate 8:2): $R_{\mathrm{f}}=0.3\left(\mathrm{UV}, \mathrm{KMnO}_{4}\right) ;{ }^{1} \mathrm{H} \mathrm{NMR}\left(400 \mathrm{MHz}, \mathrm{CDCl}_{3}\right): \delta 8.76$ (s, 0.7H), $8.29(\mathrm{~s}, 1 \mathrm{H}), 8.08(\mathrm{~d}, J=8.0 \mathrm{~Hz}, 0.7 \mathrm{H}), 7.95-7.93(\mathrm{~m}, 2.7 \mathrm{H}), 7.84-7.69(\mathrm{~m}, 9.5 \mathrm{H})$, $7.61-7.44(\mathrm{~m}, 5.1 \mathrm{H}), 7.30(\mathrm{t}, J=7.5 \mathrm{~Hz}, 0.7 \mathrm{H}), 3.91(\mathrm{~s}, 2.1 \mathrm{H}), 3.87(\mathrm{~s}, 3 \mathrm{H}) ;{ }^{13} \mathrm{C} \mathrm{NMR}(100 \mathrm{MHz}$, $\left.\mathrm{CDCl}_{3}\right): \delta 166.9,166.8,164.0,163.7,143.1,142.4,134.5,134.3,134.0,133.4,133.0,132.2,132.0$, $131.4,131.2,130.3,130.0,129.9,128.8,128.7$, 128.6, 127.7, 127.6, 127.0, 126.7, 126.5, 125.5, 125.2, 124.7, 124.3, 124.1, 123.8, 122.6, 120.0, 53.0, 52.9; IR (film): 1782, $1720 \mathrm{~cm}^{-1}$; MS (ESI): $\mathrm{m} / \mathrm{z}=358[\mathrm{M}+1] ; \mathrm{C}_{22} \mathrm{H}_{15} \mathrm{NO}_{4}$ (357.10): calcd. C, 73.94; H, 4.23; N, 3.92; found C, 74.19; H, 4.29; $\mathrm{N}, 3.87$.

Methyl (Z)-3-(2,3-/3,4-dimethylphenyl)-2-(1,3-dioxoisoindolin-2-yl)acrylate (5i)<smiles>COC(=O)C(Nc1ccccc1)c1ccc(C)c(C)c1</smiles> 
The title compound was prepared according to the general procedure using $o$-xylene $(0.3 \mathrm{~mL}, 2.5$ mmol). The product was purified by flash chromatography (cyclohexane/ ethyl acetate 7:3) to give $\mathbf{5 i}$ (94 mg, as a mixture of product isomers, 94\% yield, 1:3.5 $\alpha: \beta)$ as off-white solid.

TLC (cyclohexane/ ethyl acetate 7:3): $R_{\mathrm{f}}=0.46\left(\mathrm{UV}, \mathrm{KMnO}_{4}\right) ;{ }^{1} \mathrm{H} \mathrm{NMR}\left(400 \mathrm{MHz}, \mathrm{CDCl}_{3}\right): \delta 8.33$ (s, 0.28H), $8.07(\mathrm{~s}, 1 \mathrm{H}), 7.93(\mathrm{dd}, J=5.5,3.0 \mathrm{~Hz}, 2 \mathrm{H}), 7.82(\mathrm{dd}, J=5.5,3.0 \mathrm{~Hz}, 0.56 \mathrm{H}), 7.79(\mathrm{dd}, J$ $=5.5,3.0 \mathrm{~Hz}, 2 \mathrm{H}), 7.72(\mathrm{dd}, J=5.5,3.0 \mathrm{~Hz}, 0.56 \mathrm{H}), 7.18(\mathrm{~s}, 1 \mathrm{H}), 7.15(\mathrm{~d}, J=7.5 \mathrm{~Hz}, 1 \mathrm{H}), 7.06(\mathrm{~d}$, $J=7.5 \mathrm{~Hz}, 0.28 \mathrm{H}), 7.03(\mathrm{~d}, J=7.5 \mathrm{~Hz}, 1 \mathrm{H}), 6.98(\mathrm{~d}, J=7.5 \mathrm{~Hz}, 0.28 \mathrm{H}), 6.89$ (t, $J=7.5 \mathrm{~Hz}$, $0.28 \mathrm{H}), 3.85(\mathrm{~s}, 0.84 \mathrm{H}), 3.82(\mathrm{~s}, 3 \mathrm{H}), 2.32(\mathrm{~s}, 0.84 \mathrm{H}), 2.27(\mathrm{~s}, 0.84 \mathrm{H}), 2.20(\mathrm{~s}, 3 \mathrm{H}), 2.14(\mathrm{~s}, 3 \mathrm{H}) ;{ }^{13} \mathrm{C}$ NMR (100 MHz, $\left.\mathrm{CDCl}_{3}\right): \delta 166.9,166.8,164.1,163.7,144.4,143.2,140.0,137.1,135.8,134.4$, $134.2,132.3,132.2,132.0,131.4,131.2,130.3,129.9,126.6,125.6,124.7,124.0,123.8,123.5$, 121.6, 118.7, 52.84, 52.77, 20.2, 19.8, 19.7, 16.3; IR (film): 1785, $1721 \mathrm{~cm}^{-1}$; MS (ESI): m/z =336 $[\mathrm{M}+1], 304$ [M - 31]; $\mathrm{C}_{20} \mathrm{H}_{17} \mathrm{NO}_{4}$ (335.12): calcd. C, 71.63; H, 5.11; N, 4.18; found $\mathrm{C}, 71.81 ; \mathrm{H}$, $5.06 ; \mathrm{N}, 4.23$.

\section{Methyl (Z)-3-(2,4-/3,5-dimethylphenyl)-2-(1,3-dioxoisoindolin-2-yl)acrylate (5j)}<smiles>CC(=O)C(Nc1ccc(C)cc1)=PC1CCCCC1</smiles>

The title compound was prepared according to the general procedure using $m$-xylene $(0.3 \mathrm{~mL}, 2.45$ mmol). The product was purified by flash chromatography (cyclohexane/ ethyl acetate 9:1) to give 5j (85 mg, as a mixture of product isomers, $85 \%$ yield, $2: 1 \alpha: \beta)$ as off-white solid.

TLC (cyclohexane/ ethyl acetate 9:1): $R_{\mathrm{f}}=0.18\left(\mathrm{UV}, \mathrm{KMnO}_{4}\right) ;{ }^{1} \mathrm{H} \mathrm{NMR}\left(400 \mathrm{MHz}, \mathrm{CDCl}_{3}\right): \delta 8.26$ (s, 1H), 8.06 (s, 0.5H), $7.92(\mathrm{dd}, J=5.5,3.0 \mathrm{~Hz}, 1 \mathrm{H}), 7.85$ (dd, $J=5.5,3.0 \mathrm{~Hz}, 2 \mathrm{H}), 7.79$ (dd, $J=$ 5.5, 3.0 Hz, 1H), $7.73(\mathrm{dd}, J=5.5,3.0 \mathrm{~Hz}, 2 \mathrm{H}), 7.06(\mathrm{~d}, J=8.0 \mathrm{~Hz}, 1 \mathrm{H}), 7.01-7.00(\mathrm{~m}, 2 \mathrm{H}), 6.95$ (s, 0.5H), $6.79(\mathrm{~d}, J=8.0 \mathrm{~Hz}, 1 \mathrm{H}), 3.83(\mathrm{~s}, 3 \mathrm{H}), 3.82(\mathrm{~s}, 1.5 \mathrm{H}), 2.40(\mathrm{~s}, 3 \mathrm{H}), 2.22(\mathrm{~s}, 3 \mathrm{H}), 2.17(\mathrm{~s}$, $3 \mathrm{H}) ;{ }^{13} \mathrm{C} \mathrm{NMR}\left(100 \mathrm{MHz}, \mathrm{CDCl}_{3}\right): \delta 166.9,166.8,164.0,163.9,143.4,142.6,140.1,138.3,137.7$, $134.5,134.3,132.5,132.2$, 132.1, 132.0, 131.3, 129.1, 127.2, 126.85, 126.83, 123.9, 123.8, 120.6, 119.5, 52.8, 52.8, 21.24, 21.18, 19.9; IR (film): 1781, $1720 \mathrm{~cm}^{-1}$; MS (ESI): m/z =336 [M + 1], 304 [M - 31]; $\mathrm{C}_{20} \mathrm{H}_{17} \mathrm{NO}_{4}$ (335.12): calcd. C, 71.63; H, 5.11; N, 4.18; found C, 71.43; H, 5.03; N, 4.12.

\section{Methyl (Z)-3-(2,3-/3,4-dichlorophenyl)-2-(1,3-dioxoisoindolin-2-yl)acrylate (5k)}


<smiles>CC(=O)C([PH2+])=C1C=C(Cl)C(Cl)=CP1</smiles>

The title compound was prepared according to the general procedure using 1,2-dichlorobenzene $(1.1 \mathrm{~mL}, 9.6 \mathrm{mmol})$. The product was purified by flash chromatography (cyclohexane/ ethyl acetate $8: 2)$ to give $5 \mathbf{k}(82 \mathrm{mg}$, as a mixture of product isomers, $73 \%$ yield, $1: 1.6 \alpha: \beta)$ as white solid.

TLC (cyclohexane/ ethyl acetate 8:2): $R_{\mathrm{f}}=0.36\left(\mathrm{UV}, \mathrm{KMnO}_{4}\right) ;{ }^{1} \mathrm{H} \mathrm{NMR}\left(400 \mathrm{MHz}, \mathrm{CDCl}_{3}\right): \delta 8.25$ (s, 0.6H), $7.99(\mathrm{~s}, 1 \mathrm{H}), 7.93(\mathrm{dd}, J=5.5,3.0 \mathrm{~Hz}, 2 \mathrm{H}), 7.86(\mathrm{dd}, J=5.5,3.0 \mathrm{~Hz}, 1.2 \mathrm{H}), 7.81$ (dd, $J=$ 5.5, 3.0 Hz, 2H), $7.76(\mathrm{dd}, J=5.5,3.0 \mathrm{~Hz}, 1.2 \mathrm{H}), 7.48$ (d, $J=1.5 \mathrm{~Hz}, 1 \mathrm{H}), 7.39$ (dd, $J=8.0,1.0 \mathrm{~Hz}$, $0.6 \mathrm{H}), 7.36(\mathrm{~d}, J=8.5 \mathrm{~Hz}, 1 \mathrm{H}), 7.22(\mathrm{dd}, J=8.5,1.5 \mathrm{~Hz}, 1 \mathrm{H}), 7.17(\mathrm{dd}, J=8.0,1.0 \mathrm{~Hz}, 0.6 \mathrm{H}), 7.03$ $(\mathrm{t}, J=8.0 \mathrm{~Hz}, 0.6 \mathrm{H}), 3.87(\mathrm{~s}, 1.8 \mathrm{H}), 3.83(\mathrm{~s}, 3 \mathrm{H}) ;{ }^{13} \mathrm{C} \mathrm{NMR}\left(100 \mathrm{MHz}, \mathrm{CDCl}_{3}\right): \delta 166.6,166.4$, $163.4,163.2,140.2,139.8,134.7,134.61,134.56,134.3,133.8,133.7,133.2,132.3,131.93$, 131.86, 131.5, 131.2, 131.0, 127.8, 127.4, 126.6, 124.2, 124.0, 123.6, 121.9, 53.15, 53.11; IR (film): 1789, $1723 \mathrm{~cm}^{-1}$; MS (ESI): $\mathrm{m} / \mathrm{z}=376[\mathrm{M}+1], 344$ [M - 31]; $\mathrm{C}_{18} \mathrm{H}_{11} \mathrm{Cl}_{2} \mathrm{NO}_{4}$ (375.01): calcd. C, 57.47; H, 2.95; N, 3.72; found C, 57.63; H, 2.91; N, 3.81.

\section{Methyl (Z)-2-(1,3-dioxoisoindolin-2-yl)-3-(2-/3-/4-(trifluoromethyl)phenyl)acrylate (5l)}<smiles>CC(=O)c1ccccc1N=C(c1ccccc1)c1ccccc1</smiles>

The title compound was prepared according to the general procedure using (trifluoromethyl)benzene $(1.1 \mathrm{~mL}, 9 \mathrm{mmol})$. The product was purified by flash chromatography (cyclohexane/ ethyl acetate 8:2) to give 51 (60 mg, 53\% yield, indistinguishable mixture of $o: m: p)$ as white solid.

TLC (cyclohexane/ ethyl acetate 8:2): $R_{\mathrm{f}}=0.44\left(\mathrm{UV}, \mathrm{KMnO}_{4}\right) ;{ }^{1} \mathrm{H}$ NMR $\left(400 \mathrm{MHz}, \mathrm{CDCl}_{3}\right): \delta 8.09$ - $7.99(\mathrm{~m}, 1 \mathrm{H}), 7.83(\mathrm{dd}, J=5.5,3.0 \mathrm{~Hz}, 2 \mathrm{H}), 7.78(\mathrm{dd}, J=5.5,3.0 \mathrm{~Hz}, 0.3 \mathrm{H}), 7.71(\mathrm{dd}, J=5.5,3.0$ $\mathrm{Hz}, 2 \mathrm{H}), 7.67(\mathrm{dd}, J=5.5,3.0 \mathrm{~Hz}, 0.3 \mathrm{H}), 7.56-7.34(\mathrm{~m}, 6 \mathrm{H}), 3.76(\mathrm{~s}, 3 \mathrm{H}) ;{ }^{13} \mathrm{C} \mathrm{NMR}(100 \mathrm{MHz}$, $\left.\mathrm{CDCl}_{3}\right): \delta 171.8,168.4,166.7,166.6,163.5,163.4,141.3,141.2,136.6,135.9,134.7,134.3,133.8$, 133.2, 132.6, 131.9 - 131.2 (m), 130.2, 129.5, 129.31, 129.29, 128.5, 126.9 (q, $J=4 \mathrm{~Hz}), 126.2$ (q, $J=4 \mathrm{~Hz}), 125.8$ (q, $J=4 \mathrm{~Hz}), 124.2,124.1,123.6,122.0,119.4,117.7,53.13,53.11$; IR (film): 1785, $1721 \mathrm{~cm}^{-1} ;{ }^{19} \mathrm{~F}$ NMR $\left(377 \mathrm{MHz}, \mathrm{CDCl}_{3}\right): \delta-63.3,-63.4,-63.5 ; \mathrm{MS}(\mathrm{ESI}): \mathrm{m} / \mathrm{z}=376[\mathrm{M}+1]$, $345[\mathrm{M}-31]$.

Ethyl (Z)-2-/3-/4-(2-(1,3-dioxoisoindolin-2-yl)-3-methoxy-3-oxoprop-1-enyl)benzoate (5m) 


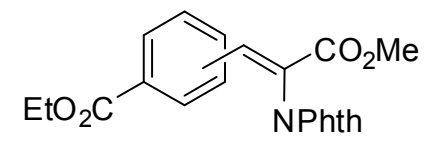

The title compound was prepared according to the general procedure using ethyl benzoate $(1.1 \mathrm{~mL}$, $7.5 \mathrm{mmol}$ ). The product was purified by flash chromatography (cyclohexane/ ethyl acetate 8:2) to give 5m (90 mg, as a mixture of product isomers, 79\% yield, 1:9:2.6 o:m:p) as white solid.

TLC (cyclohexane/ ethyl acetate 8:2): $R_{\mathrm{f}}=0.3\left(\mathrm{UV}, \mathrm{KMnO}_{4}\right) ;{ }^{1} \mathrm{H} \mathrm{NMR}\left(400 \mathrm{MHz}, \mathrm{CDCl}_{3}\right): \delta 8.14$ (s, 1H), $8.11(\mathrm{~m}, 1.5 \mathrm{H}), 8.00(\mathrm{~d}, J=7.5 \mathrm{~Hz}, 0.7 \mathrm{H}), 7.96(\mathrm{~d}, J=8.0 \mathrm{~Hz}, 0.5 \mathrm{H}), 7.94-7.90(\mathrm{~m}$, 2.6H), $7.86(\mathrm{dd}, J=5.5,3.0 \mathrm{~Hz}, 0.4 \mathrm{H}), 7.80(\mathrm{dd}, J=5.5,3.0 \mathrm{~Hz}, 2.6 \mathrm{H}), 7.75(\mathrm{dd}, J=5.5,3.0 \mathrm{~Hz}$, $0.4 \mathrm{H}), 7.60-7.57(\mathrm{~m}, 1 \mathrm{H}), 7.49-7.44(\mathrm{~m}, 1.2 \mathrm{H}), 7.38(\mathrm{t}, J=8.0 \mathrm{~Hz}, 1 \mathrm{H}), 4.43(\mathrm{q}, J=7.0 \mathrm{~Hz}$, $0.22 \mathrm{H}), 4.32(\mathrm{q}, J=7.0 \mathrm{~Hz}, 0.6 \mathrm{H}), 4.22(\mathrm{q}, J=7.0 \mathrm{~Hz}, 2 \mathrm{H}), 3.84(\mathrm{~s}, 4.2 \mathrm{H}), 1.42(\mathrm{t}, J=7.0 \mathrm{~Hz}$, $0.3 \mathrm{H}), 1.34(\mathrm{t}, J=7.0 \mathrm{~Hz}, 0.9 \mathrm{H}), 1.18(\mathrm{t}, J=7.0 \mathrm{~Hz}, 3 \mathrm{H}) ;{ }^{13} \mathrm{C} \mathrm{NMR}\left(100 \mathrm{MHz}, \mathrm{CDCl}_{3}\right): \delta 171.2$, $168.2,166.63,166.58,165.7,165.5,163.7,163.5,145.2,141.7,136.6,134.62,134.56,134.3$, 133.6, 133.3, 132.6, 132.5, 132.3, 132.1, 132.0, 131.8, 131.4, 131.2, 130.9, 130.3, 130.1, 130.0, $129.3,129.1,129.0,128.4,128.0,124.1,124.0,123.8,123.5,122.0,121.1,61.2,61.1,53.1,53.0$, 52.9, 14.2, 14.0; IR (film): 1785, $1721 \mathrm{~cm}^{-1}$; MS (ESI): m/z =380 [M + 1], 348 [M - 31]; $\mathrm{C}_{21} \mathrm{H}_{17} \mathrm{NO}_{6}$ (379.11): calcd. C, 66.49; H, 4.52; N, 3.69; found C, 66.64; H, 4.49; N, 3.65.

\section{(Z)-Methyl 3-(2-/3-/4-acetylphenyl)-2-(1,3-dioxoisoindolin-2-yl)acrylate (5n)}

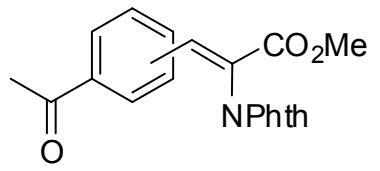

The title compound was prepared according to the general procedure using acetophenone $(1.1, \mathrm{~mL}$, $9.4 \mathrm{mmol}$ ). The product was purified by flash chromatography (cyclohexane/ ethyl acetate 7:3) to give 5n (43 mg, as a mixture of product isomers, 41\% yield, , 1:3.7 o:m) as white solid.

TLC (cyclohexane/ ethyl acetate 8:2): $R_{\mathrm{f}}=0.18\left(\mathrm{UV}, \mathrm{KMnO}_{4}\right) ;{ }^{1} \mathrm{H} \mathrm{NMR}\left(400 \mathrm{MHz}, \mathrm{CDCl}_{3}\right): \delta 8.16$ $(\mathrm{s}, 1 \mathrm{H}), 8.13(\mathrm{~s}, 0.3 \mathrm{H}), 8.00-7.99(\mathrm{~m}, 1 \mathrm{H}), 7.94-7.87(\mathrm{~m}, 4.2 \mathrm{H}), 7.81(\mathrm{dd}, J=5.5,3.0 \mathrm{~Hz}, 2.6 \mathrm{H})$, 7.49 (d, $J=7.5 \mathrm{~Hz}, 1 \mathrm{H}), 7.49$ (d, $J=8.0 \mathrm{~Hz}, 0.6 \mathrm{H}), 7.41$ (t, $J=7.5 \mathrm{~Hz}, 1 \mathrm{H}), 3.86$ (s, 3.9H), 2.56 (s, 0.9H), 2.45 (s, 3H); ${ }^{13} \mathrm{C} \mathrm{NMR}\left(100 \mathrm{MHz}, \mathrm{CDCl}_{3}\right): \delta 197.1,196.9,166.7,166.6,163.6,163.5,141.8$, 141.5 , 137.9, 137.5, 136.9, 134.68, 134.66, 133.2, 132.8, 132.0, 129.9, 129.36, 129.34, 129.2, 128.7, 124.14, 124.09, 122.1, 121.4, 53.09, 53.06, 26.6, 26.4; IR (film): 1787, $1720 \mathrm{~cm}^{-1}$; MS (ESI): $\mathrm{m} / \mathrm{z}=350[\mathrm{M}+1], 319[\mathrm{M}-31] ; \mathrm{C}_{20} \mathrm{H}_{15} \mathrm{NO}_{5}$ (349.10): calcd. C, 68.76; H, 4.33; N, 4.01; found C, 68.94; H, 4.40; N, 3.96 . 
Procedure for large scale synthesis of compound 5f. Methyl 2-(1,3-dioxoisoindolin-2-yl)acrylate (1f) (1 g, $4.3 \mathrm{mmol}), 1,3,5$-trifluorobenzene (4.3 mL, $41.7 \mathrm{mmol}), \operatorname{Pd}(\mathrm{OAc})_{2}(48 \mathrm{mg}, 0.21 \mathrm{mmol})$, 3,5-dichloropyridine $(31 \mathrm{mg}, 0.21 \mathrm{mmol})$, tert-butyl peroxybenzoate $(0.82 \mathrm{~mL}, 4.3 \mathrm{mmol})$, and acetic acid $(20 \mathrm{~mL})$ were combined in a $50 \mathrm{~mL}$ Schlenk equipped with a magnetic stir bar. The reaction was stirred at $100{ }^{\circ} \mathrm{C}$ for $16 \mathrm{~h}$. The resulting reaction mixture was diluted with ethyl acetate $(50 \mathrm{~mL})$ and quenched with a $10 \%$ aqueous $\mathrm{Na}_{2} \mathrm{SO}_{3}$ solution $(50 \mathrm{~mL})$. The organic layer was separated and washed with $10 \%$ aqueous $\mathrm{Na}_{2} \mathrm{SO}_{3}$ solution $(1 \times 50 \mathrm{~mL})$, saturated aqueous $\mathrm{NaHCO}_{3}$ solution $(3 \times 50 \mathrm{~mL})$, and brine $(1 \times 50 \mathrm{~mL})$. The organic layer was dried over $\mathrm{Na}_{2} \mathrm{SO}_{4}$, filtered, and concentrated under reduced pressure. The product was purified by flash chromatography (gradient from cyclohexane/ ethyl acetate 9:1 to cyclohexane/ ethyl acetate 8:2) to give $\mathbf{5 f}(930 \mathrm{mg}, 60 \%)$ as white solid.

\section{Methyl 2-(1,3-dioxoisoindolin-2-yl)-3-hydroxy-3-(2,4,6-trifluorophenyl)propanoate (7)}<smiles>CC(=O)C([NH+]c1ccccc1)[C@H](O)c1c(F)cc(F)cc1F</smiles>

A mixture of $\mathrm{CuCl}(1 \mathrm{mg}, \quad 0.01 \mathrm{mmol}), \quad \mathrm{KO} t \mathrm{Bu} \quad(3.5 \mathrm{mg}, 0.03 \mathrm{mmol})$, and 1,2bis(diphenylphosphanyl)benzene (dppbz; $5 \mathrm{mg}, 0.01 \mathrm{mmol})$ in anhydrous THF $(0.12 \mathrm{~mL})$ was stirred for $30 \mathrm{~min}$ in a sealed tube. $\mathrm{B}_{2} \mathrm{pin}_{2}(66 \mathrm{mg}, 0.26 \mathrm{mmol})$ in THF $(0.12 \mathrm{~mL})$ was added. The reaction mixture was stirred for $10 \mathrm{~min}$, and then methyl (Z)-2-(1,3-dioxoisoindolin-2-yl)-3-(2,4,6trifluorophenyl)acrylate (7f) $(72 \mathrm{mg}, 0.2 \mathrm{mmol})$ in THF $(0.12 \mathrm{~mL})$ was added to the reaction mixture, followed by $\mathrm{MeOH}(16 \mu \mathrm{L}, 0.4 \mathrm{mmol})$. The reaction mixture was stirred at the room temperature for $20 \mathrm{~h}$, and then it was filtered through a short plug of silica gel. The solvents were evaporated under reduced pressure to give 6 as a mixture of two diastereoisomers (2:1). The product obtained was used for the following reaction without further purification.

To a solution of methyl 2-(1,3-dioxoisoindolin-2-yl)-3-(4,4,5,5-tetramethyl-1,3,2-dioxaborolan-2yl)-3-(2,4,6-trifluorophenyl)propanoate (6) $(0.2 \mathrm{mmol})$ in THF $(1 \mathrm{~mL})$ and $\mathrm{H}_{2} \mathrm{O}(1 \mathrm{~mL})$ was added sodium perborate $(77 \mathrm{mg}, 0.5 \mathrm{mmol})$. The reaction mixture was stirred vigorously at room temperature for $3 \mathrm{~h}$. The mixture was diluted with $\mathrm{H}_{2} \mathrm{O}(3 \mathrm{~mL})$ and extracted with EtOAc $(3 \times 8$ $\mathrm{mL}$ ). The combined organic layer were washed with brine, dried over $\mathrm{Na}_{2} \mathrm{SO}_{4}$, filtered, and concentrated under reduced pressure. The residue was purified by flash chromatography (cyclohexane/ ethyl acetate 8:2) to give 7 (36 mg, 47\% yield, over two steps) as white solid. 
TLC (cyclohexane/ ethyl acetate 8:2): $R_{\mathrm{f}}=0.24\left(\mathrm{UV}, \mathrm{KMnO}_{4}\right) ;{ }^{1} \mathrm{H} \mathrm{NMR}\left(400 \mathrm{MHz}, \mathrm{CDCl}_{3}\right): \delta 7.89$ $(\mathrm{dd}, J=5.5,3.0 \mathrm{~Hz}, 2 \mathrm{H}), 7.80-7.72(\mathrm{~m}, 3 \mathrm{H}), 7.72(\mathrm{dd}, J=5.5,3.0 \mathrm{~Hz}, 1 \mathrm{H}), 6.62(\mathrm{t}, J=8.5 \mathrm{~Hz}$, 2H), $6.55(\mathrm{t}, J=8.5 \mathrm{~Hz}, 1 \mathrm{H}), 5.95-5.91(\mathrm{~m}, 1.5 \mathrm{H}), 5.39-5.30(\mathrm{~m}, 1.5 \mathrm{H}), 4.27(\mathrm{~d}, J=3.0 \mathrm{~Hz}$, $0.5 \mathrm{H}), 4.06(\mathrm{~d}, J=9.5 \mathrm{~Hz}, 1 \mathrm{H}), 3.80(\mathrm{~s}, 1.5 \mathrm{H}), 3.78(\mathrm{~s}, 3 \mathrm{H}) ;{ }^{13} \mathrm{C} \mathrm{NMR}\left(100 \mathrm{MHz}, \mathrm{CDCl}_{3}\right): \delta 170.4$, 168.2, 167.0, 166.6, 162.7 (dt, $J=249,15 \mathrm{~Hz}), 162.4$ (dt, $J=249,15 \mathrm{~Hz}), 161.8$ (ddd, $J=249,15$, $10 \mathrm{~Hz}), 161.1$ (ddd, $J=249,15,10 \mathrm{~Hz}), 134.6,134.5,131.5,131.2,123.9,123.8,112.6(\mathrm{td}, J=15$, $4 \mathrm{~Hz}), 110.4(\mathrm{td}, J=15,4 \mathrm{~Hz}), 101.0-100.3(\mathrm{~m}), 65.4,63.6,56.7,53.4,53.3,53.1 ;{ }^{19} \mathrm{~F}$ NMR $(377$ $\mathrm{MHz}, \mathrm{CDCl}_{3}$ ): $\delta-106.6$ (quin, $J=8.5 \mathrm{~Hz}, 0.5 \mathrm{~F}$ ), -107.5 (quin, $J=8.5 \mathrm{~Hz}, 1 \mathrm{~F}$ ), -109.1 (t, $J=8.5 \mathrm{~Hz}$, 1F), -111.1 (t, $J=8.5 \mathrm{~Hz}, 2 \mathrm{~F})$; IR (film): 3485, 1775, $1720 \mathrm{~cm}^{-1}$; MS (ESI): $\mathrm{m} / \mathrm{z}=380[\mathrm{M}+1]$; $\mathrm{C}_{18} \mathrm{H}_{12} \mathrm{~F}_{3} \mathrm{NO}_{5}$ (379.07): calcd. C, 57.00; H, 3.19; N, 3.69; found C, 56.78; H, 3.25; N, 3.76.

Methyl

\section{trifluorophenyl)propanoate (8)}<smiles>COC(=O)C(C(C)c1ccccc1)C(c1ccccc1)c1c(F)cc(F)cc1F</smiles>

3-(dimethyl(phenyl)silyl)-2-(1,3-dioxoisoindolin-2-yl)-3-(2,4,6-

A mixture of $\mathrm{CuCl}(1 \mathrm{mg}, 0.01 \mathrm{mmol}), \mathrm{KO} t \mathrm{Bu}(3.5 \mathrm{mg}, 0.03 \mathrm{mmol})$, and dppbz (5 mg, $0.01 \mathrm{mmol})$ in anhydrous THF $(0.18 \mathrm{~mL})$ was stirred for $30 \mathrm{~min}$ in a sealed tube. $\mathrm{Me}_{2} \mathrm{PhSi}-\mathrm{Bpin}(71 \mu \mathrm{L}, 0.26$ mmol) was added. The reaction mixture was stirred for $10 \mathrm{~min}$, and then methyl (Z)-2-(1,3dioxoisoindolin-2-yl)-3-(2,4,6-trifluorophenyl)acrylate (5f) (72 mg, $0.2 \mathrm{mmol})$ in THF $(0.18 \mathrm{~mL})$ was added to the reaction mixture, followed by $\mathrm{MeOH}(16 \mu \mathrm{L}, 0.4 \mathrm{mmol})$. The reaction mixture was then stirred at $50{ }^{\circ} \mathrm{C}$ for $60 \mathrm{~h}$. The solvents were evaporated under reduced pressure, and the residue was purified by flash chromatography (cyclohexane/ ethyl acetate 8:2) to give 8 (60 mg, $61 \%$ yield) as a mixture of two diastereoisomers (2:1), as white solid.

TLC (cyclohexane/ ethyl acetate 8:2): $R_{\mathrm{f}}=0.41\left(\mathrm{UV}, \mathrm{KMnO}_{4}\right) ;{ }^{1} \mathrm{H}$ NMR $\left(400 \mathrm{MHz}, \mathrm{CDCl}_{3}\right): \delta 7.74$ $(\mathrm{dd}, J=5.5,3.0 \mathrm{~Hz}, 1 \mathrm{H}), 7.65(\mathrm{dd}, J=5.5,3.0 \mathrm{~Hz}, 1 \mathrm{H}), 7.62-7.51(\mathrm{~m}, 5 \mathrm{H}), 7.32-7.30(\mathrm{~m}, 1.5 \mathrm{H})$, $7.24-7.22(\mathrm{~m}, 2 \mathrm{H}), 6.87-6.65(\mathrm{~m}, 5 \mathrm{H}), 6.44-6.35(\mathrm{~m}, 1 \mathrm{H}), 5.72(\mathrm{~d}, J=12.5 \mathrm{~Hz}, 1 \mathrm{H}), 5.52(\mathrm{~d}, J$ $=12.5 \mathrm{~Hz}, 0.5 \mathrm{H}), 4.18(\mathrm{~d}, J=12.5 \mathrm{~Hz}, 1.5 \mathrm{H}), 3.50(\mathrm{~s}, 4.5 \mathrm{H}), 0.49-0.47(\mathrm{~m}, 4.5 \mathrm{H}), 0.37(\mathrm{~s}, 1.5 \mathrm{H})$, $0.01(\mathrm{~s}, 3 \mathrm{H}) ;{ }^{13} \mathrm{C} \mathrm{NMR}\left(100 \mathrm{MHz}, \mathrm{CDCl}_{3}\right): \delta 169.44,169.37,167.1,166.9,162.3-161.4(\mathrm{~m})$, 159.9 - 159.0 (m), 137.2, 136.0, 134.1, 133.7, 133.6, 133.3, 131.5, 131.3, 129.1, 128.5, 127.6, 127.5, 123.4, 123.3, $113.2(\mathrm{td},, J=19,5 \mathrm{~Hz}), 112.7$ (td, , $J=19,5 \mathrm{~Hz}), 100.5$ - 99.6 (m), 52.64, 52.56, 49.8, 49.7, 24.7, 23.9, -2.4, -2.6, -2.7, -5.8; ${ }^{19} \mathrm{~F}$ NMR (377 $\left.\mathrm{MHz}, \mathrm{CDCl}_{3}\right): \delta-105.2(\mathrm{~m}, 0.5 \mathrm{~F})$, $-107.4(\mathrm{~m}, 1 \mathrm{~F}),-108.3(\mathrm{~m}, 1 \mathrm{~F}),-111.2(\mathrm{~m}, 0.5 \mathrm{~F}),-111.7(\mathrm{~m}, 0.5 \mathrm{~F}),-125.5$ (m, 1F); IR (film): 1769, 
$1716 \mathrm{~cm}^{-1}$; MS (ESI): m/z =498 [M + 1], 420 [M - 77]; $\mathrm{C}_{26} \mathrm{H}_{22} \mathrm{~F}_{3} \mathrm{NO}_{4} \mathrm{Si}$ (497.13): calcd. C, 62.77; $\mathrm{H}, 4.46 ; \mathrm{N}, 2.82$; found $\mathrm{C}, 62.57 ; \mathrm{H}, 4.37 ; \mathrm{N}, 2.88$.

\section{Methyl 2-(1,3-dioxoisoindolin-2-yl)-3-(2,4,6-trifluorophenyl)propanoate (9)}<smiles>CC(=O)C(Cc1c(F)cc(F)cc1F)[PH+]c1ccccc1</smiles>

A mixture of $\mathrm{Cu}(\mathrm{OAc})_{2} \quad(7.3 \mathrm{mg}, \quad 0.04 \mathrm{mmol})$ and ( \pm )-2,2'-bis(diphenylphosphino)-1,1'binaphthalene $(( \pm)$ BINAP, $25 \mathrm{mg}, 0.04 \mathrm{mmol})$ in anhydrous THF $(0.87 \mathrm{~mL})$ was stirred for $10 \mathrm{~min}$ in a sealed tube. Diethoxymethylsilane (DEMS, $208 \mu \mathrm{L}, 1.3 \mathrm{mmol}$ ) was added. The reaction mixture was stirred until it attained a bright lime-green color ( $\sim 5 \mathrm{~min})$, and then methyl $(Z)-2-(1,3-$ dioxoisoindolin-2-yl)-3-(2,4,6-trifluorophenyl)acrylate (5f) $(72 \mathrm{mg}, 0.2 \mathrm{mmol})$ in THF (0.34 mL) and $t \mathrm{BuOH}(124 \mu \mathrm{L}, 1.3 \mathrm{mmol})$ was added. The reaction mixture was stirred at $45^{\circ} \mathrm{C}$ for $20 \mathrm{~h}$. The solvents were evaporated under reduced pressure, and the residue was purified by flash chromatography (cyclohexane/ ethyl acetate 8:2) to give 9 (51 mg, 67\% yield) as white solid.

TLC (cyclohexane/ ethyl acetate 8:2): $R_{\mathrm{f}}=0.4\left(\mathrm{UV}, \mathrm{KMnO}_{4}\right) ;{ }^{1} \mathrm{H} \mathrm{NMR}\left(400 \mathrm{MHz}, \mathrm{CDCl}_{3}\right): \delta 7.83$ $(\mathrm{dd}, J=5.0,3.0 \mathrm{~Hz}, 2 \mathrm{H}), 7.74(\mathrm{dd}, J=5.5,3.0 \mathrm{~Hz}, 2 \mathrm{H}), 6.55(\mathrm{t}, J=7.5,2 \mathrm{H}), 5.07$ (t, $J=7.5 \mathrm{~Hz}$, $1 \mathrm{H}), 3.79(\mathrm{~s}, 3 \mathrm{H}), 3.56(\mathrm{~d}, J=7.5 \mathrm{~Hz}, 2 \mathrm{H}) ;{ }^{13} \mathrm{C} \mathrm{NMR}\left(100 \mathrm{MHz}, \mathrm{CDCl}_{3}\right): \delta 168.6,167.0,161.7(\mathrm{dt}$, $J=247,16 \mathrm{~Hz}), 161.0(\mathrm{ddd}, J=247,18,6 \mathrm{~Hz}) 134.2,131.5,123.6,108.9$ (td, $J=20,5 \mathrm{~Hz}), 100.4$ - $99.45(\mathrm{~m}), 53.0,51.0,22.2 ;{ }^{19} \mathrm{~F}$ NMR (377 MHz, $\mathrm{CDCl}_{3}$ ): $\delta-109.1$ (quin, $J=6.5 \mathrm{~Hz}, 1 \mathrm{~F}$ ), -112.3 (t, $J=6.5 \mathrm{~Hz}, 2 \mathrm{~F}$ ); IR (film): 1783, $1721 \mathrm{~cm}^{-1}$; MS (ESI): $\mathrm{m} / \mathrm{z}=364[\mathrm{M}+1] ; \mathrm{C}_{18} \mathrm{H}_{12} \mathrm{~F}_{3} \mathrm{NO}_{4}$ (363.07): calcd. C, 59.51; H, 3.33; N, 3.86; found C, 59.68; H, 3.25; N, 3.81.

\section{2-((((9H-fluoren-9-yl)methoxy)carbonyl)amino)-3-(2,4,6-trifluorophenyl)propanoic acid (10)}<smiles>O=C(O)C(Cc1c(F)cc(F)cc1F)N=CP</smiles>

The methyl 2-(1,3-dioxoisoindolin-2-yl)-3-(2,4,6-trifluorophenyl)propanoate (9) (50 mg, 0.14 $\mathrm{mmol})$ was dissolved in a 1:1 mixture of $\mathrm{DCM} / \mathrm{MeOH}(1.2 \mathrm{~mL}$ total), and ethylenediamine (56 $\mu \mathrm{l}$, $0.84 \mathrm{mmol}$ ) was added. The reaction mixture was heated to $40{ }^{\circ} \mathrm{C}$ for $3 \mathrm{~h}$, and then cooled to room temperature. The reaction mixture was stirred at room temperature for $16 \mathrm{~h}$. Then reaction mixture (with solvent) was transferred to a column with silica gel directly and purified by column chromatography (DCM/MeOH 9:1). Crude product was used directly for next step. Amino ester 
was dissolved in 1,4-dioxane $(0.6 \mathrm{~mL})$, and $\mathrm{NaHCO}_{3}$ solution $(27 \mathrm{mg}, 0.32 \mathrm{mmol}$ in $0.6 \mathrm{~mL}$ of water) was added. The mixture was cooled to $0{ }^{\circ} \mathrm{C}$ and $\mathrm{Fmoc}-\mathrm{Cl}(36 \mathrm{mg}, 0.14 \mathrm{mmol})$ was added. The ice bath was allowed to warm to room temperature for $16 \mathrm{~h}$, after which $\mathrm{H}_{2} \mathrm{O}$ and EtOAc was added to the reaction mixture. The aqueous layer was then extracted with EtOAc twice and the combined organic layers were washed with brine, dried over $\mathrm{Na}_{2} \mathrm{SO}_{4}$, filtered, and concentrated under reduced pressure. The residue was purified by flash chromatography (cyclohexane/ ethyl acetate 8:2) to give methyl 2-((((9H-fluoren-9-yl)methoxy)carbonyl)amino)-3-(2,4,6-trifluorophenyl)propanoate as white solid. ${ }^{1} \mathrm{H}$ NMR (400 MHz, $\left.\mathrm{CDCl}_{3}\right): \delta 7.78$ (d, $\left.J=7.5 \mathrm{~Hz}, 2 \mathrm{H}\right), 7.58$ (t, $\left.J=6.6 \mathrm{~Hz}, 2 \mathrm{H}\right), 7.42$ (t, $J=7.5 \mathrm{~Hz}, 2 \mathrm{H}), 7.33$ (t, $J=7.5,1 \mathrm{H}), 7.32$ (t, $J=7.5,1 \mathrm{H}), 6.65(\mathrm{t}, J=7.5 \mathrm{~Hz}, 2 \mathrm{H}), 5.42(\mathrm{~d}, J=8.5$ $\mathrm{Hz}, 1 \mathrm{H}), 4.67(\mathrm{~m}, 1 \mathrm{H}), 4.41(\mathrm{dd}, J=10.5,7.0 \mathrm{~Hz}, 1 \mathrm{H}), 4.29$ (dd, $J=10.5,7.0 \mathrm{~Hz}, 1 \mathrm{H}), 4.20(\mathrm{t}, J=$ $7.0 \mathrm{~Hz}, 1 \mathrm{H}), 3.79$ (s, 3H), 3.30 (dd, $J=14.0,5.5 \mathrm{~Hz}, 1 \mathrm{H}), 3.07(\mathrm{dd}, J=14.0,7.0 \mathrm{~Hz}, 1 \mathrm{H}) ;{ }^{13} \mathrm{C}$ NMR $\left(100 \mathrm{MHz}, \mathrm{CDCl}_{3}\right): \delta 171.6,161.85(\mathrm{dt}, J=247,16 \mathrm{~Hz}), 161.81(\mathrm{dt}, J=247,16 \mathrm{~Hz}), 1438,143.7$, 141.3, 127.7, 127.0, 125.10, 125.07, 120.0, 108.1 (dt, $J=20,5 \mathrm{~Hz}), 100.1$ (dt, $J=28,2 \mathrm{~Hz}), 67.2$, 53.2, 52.7, 47.0, 25.4; MS (ESI): $\mathrm{m} / \mathrm{z}=456[\mathrm{M}+1]$. Carboxylic ester (54 mg, $0.12 \mathrm{mmol})$ was dissolved in 1,2-dichloroethane $(0.6 \mathrm{~mL})$, and trimethyltin hydroxide $(43 \mathrm{mg}, 0.24 \mathrm{mmol})$ was added. The mixture was heated at $80{ }^{\circ} \mathrm{C}$ for $2 \mathrm{~h}$. When the reaction was complete, the mixture was concentrated in vacuo, and the residue was dissolved in ethyl acetate $(1 \mathrm{~mL})$. The organic layer was washed with $1 \mathrm{~N} \mathrm{KHSO}_{4}(10 \mathrm{~mL})$, brine $(10 \mathrm{~mL})$, dried over $\mathrm{Na}_{2} \mathrm{SO}_{4}$, filtered, and concentrated under reduced pressure. The residue was purified by flash chromatography (DCM/MeOH 9:1) to give $\mathbf{1 0}$ (35 mg, 56\% yield over three steps) as white solid.

TLC (DCM/MeOH 9:1): $R_{\mathrm{f}}=0.3\left(\mathrm{UV}, \mathrm{KMnO}_{4}\right) ;{ }^{1} \mathrm{H}$ NMR $\left(400 \mathrm{MHz}, \mathrm{CD}_{3} \mathrm{OD}\right) \delta 7.79(\mathrm{~d}, J=7.5$ $\mathrm{Hz}, 2 \mathrm{H}), 7.60(\mathrm{~d}, J=7.5 \mathrm{~Hz}, 2 \mathrm{H}), 7.41-7.37$ (m, 2H), $7.32-7.28(\mathrm{~m}, 2 \mathrm{H}), 6.77$ (t, $J=8.0 \mathrm{~Hz}$, 2H), $4.39(\mathrm{dd}, J=9.5,5.0 \mathrm{~Hz}, 1 \mathrm{H}), 4.26(\mathrm{dd}, J=10.0,6.5 \mathrm{~Hz}, 1 \mathrm{H}), 4.18-4.10(\mathrm{~m}, 2 \mathrm{H}), 3.27(\mathrm{dd}, J$ $=14.0,5.0 \mathrm{~Hz}, 1 \mathrm{H}), 3.04(\mathrm{dd}, J=14.0,9.5 \mathrm{~Hz}, 1 \mathrm{H}) ;{ }^{13} \mathrm{C} \mathrm{NMR}\left(100 \mathrm{MHz}, \mathrm{CD}_{3} \mathrm{OD}\right) \delta 175.5,161.8$ $(\mathrm{dm}, J=255 \mathrm{~Hz}), 161.6(\mathrm{dm}, J=255 \mathrm{~Hz}), 143.8,141.1,127.3,126.7,124.85,124.78,119.5,110.0$, 109.9, 66.6, 54.5, 46.9, 25.0; ${ }^{19} \mathrm{~F}$ NMR (377 MHz, $\mathrm{CDCl}_{3}$ ): $\delta-112.6$ (quin, $J=8.5 \mathrm{~Hz}, 1 \mathrm{~F}$ ), -113.3 (t, $J=8.5 \mathrm{~Hz}, 2 \mathrm{~F}) ;$ m.p. $156-158{ }^{\circ} \mathrm{C}$; IR (film): $3434,1721,1690 \mathrm{~cm}^{-1}$; MS (ESI): m/z = $442[\mathrm{M}+$ 1]; $\mathrm{C}_{24} \mathrm{H}_{18} \mathrm{~F}_{3} \mathrm{NO}_{4}$ (441.12): calcd. C, 65.31; H, 4.11; N, 3.17; found C, 65.58; H, 4.15; N, 3.14 .

\section{References:}

(1) C. Prashant, B. Sanjay Synthetic Commun. 2015, 45, 391-402.

(2) S. Koch, D. Schollmeyer, H. Löwe, H. Kunz Chem. Eur. J. 2013, 19, 7020-7041

(3) C. Xie, D. Han, J. Liu, T. Xie Synlett. 2009, 3155-3158.

(4) B. M. Trost, G. R. Dake J. Am. Chem. Soc. 1997, 119, 7595-7596.

(5) N. Yoshitomo, S. Makoto PCT Int. Appl. (2015), WO 2015083744

(6) Easton, C. J.; Hutton, C. A.; Roselt, P. D.; Tiekink, E. R. T. Tetrahedron 1994, 50, 7327 
Methyl 2-(2-oxopyrrolidin-1-yl)acrylate (1d)
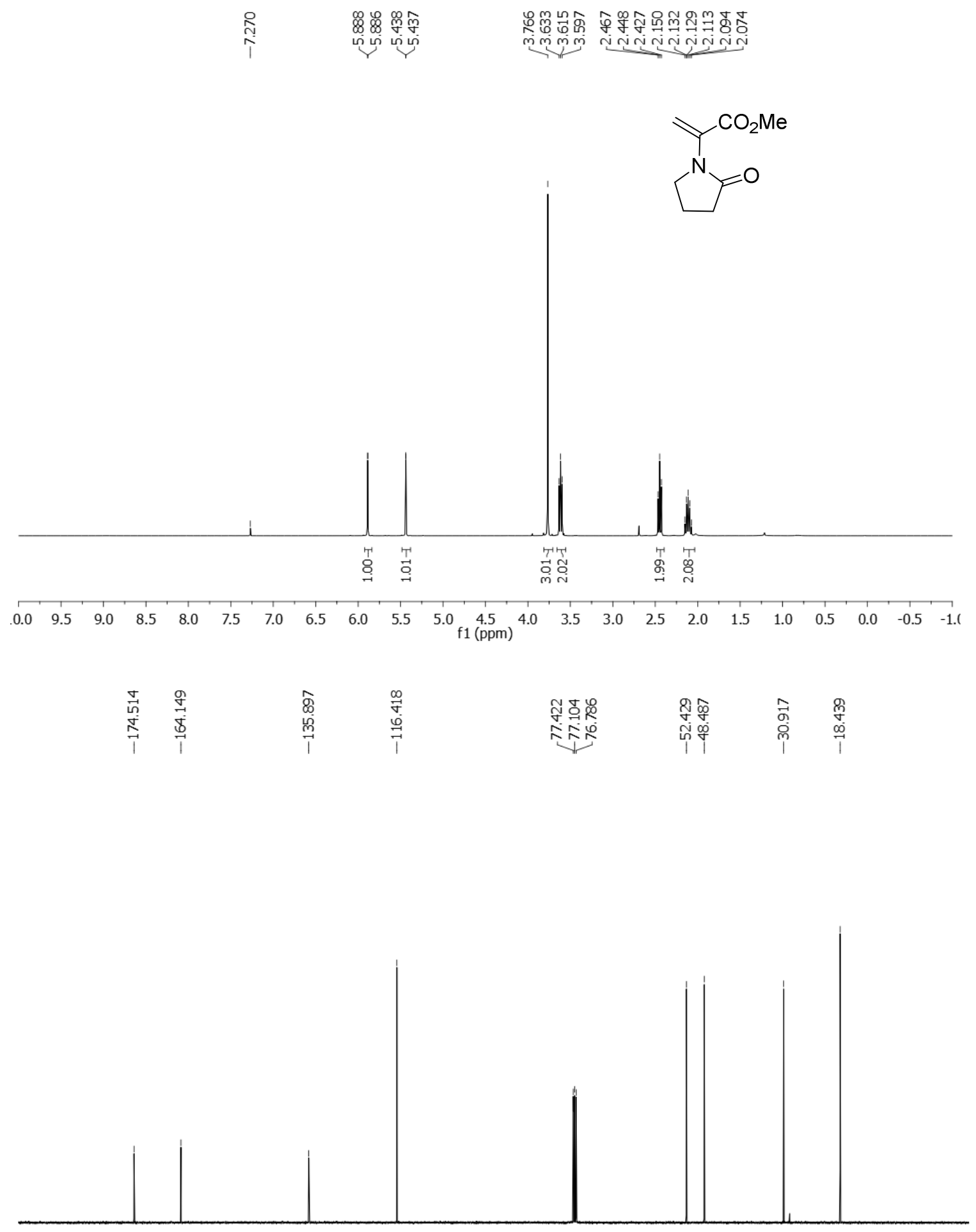

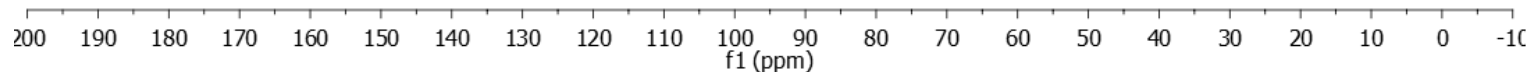




\section{Methyl (Z)-2-(2-oxopyrrolidin-1-yl)-3-phenylacrylate (3a)}

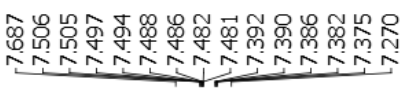

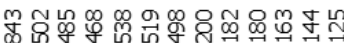

minm NiviNivin
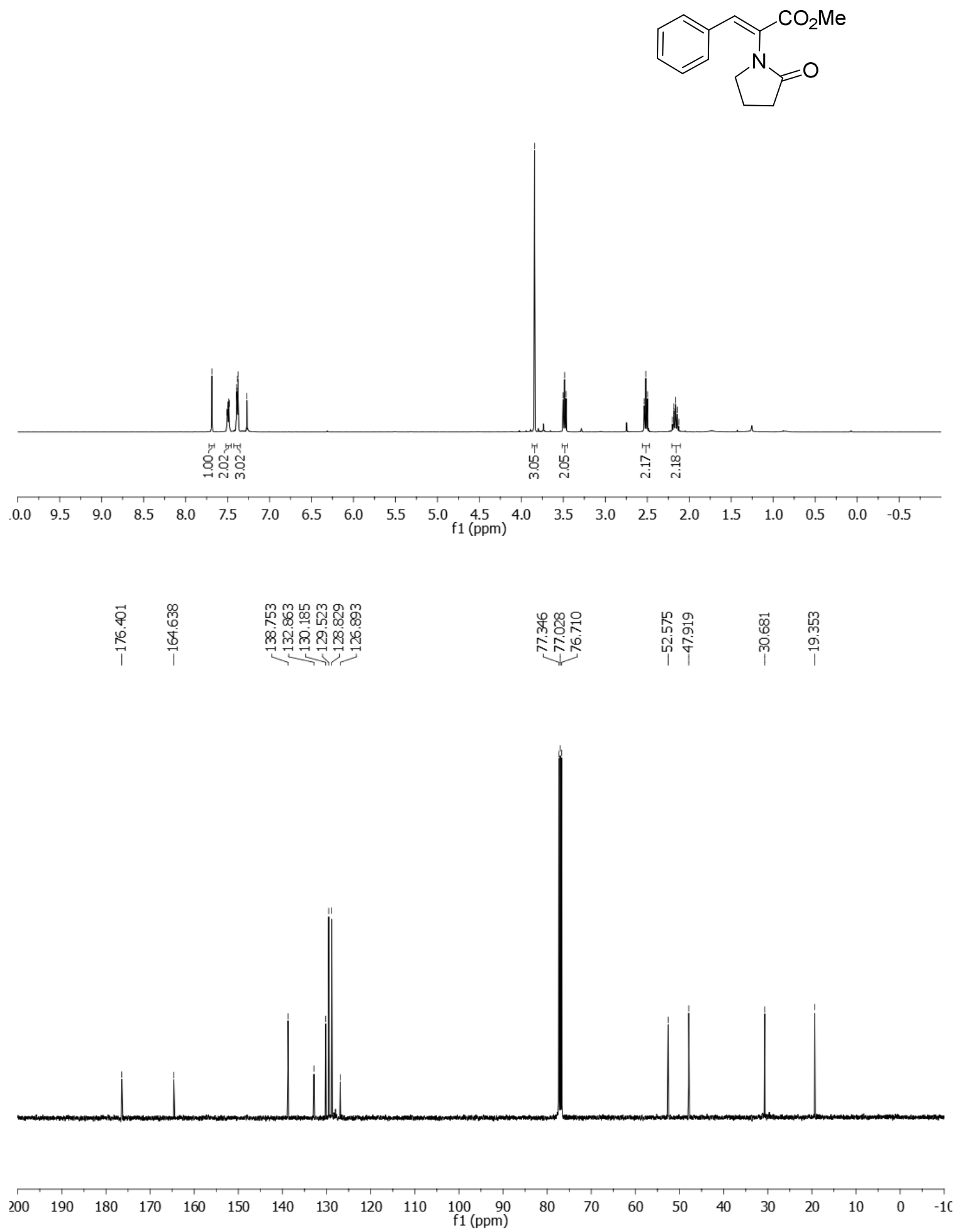
Methyl (Z)-2-(2-oxooxazolidin-3-yl)-3-phenylacrylate (4a)

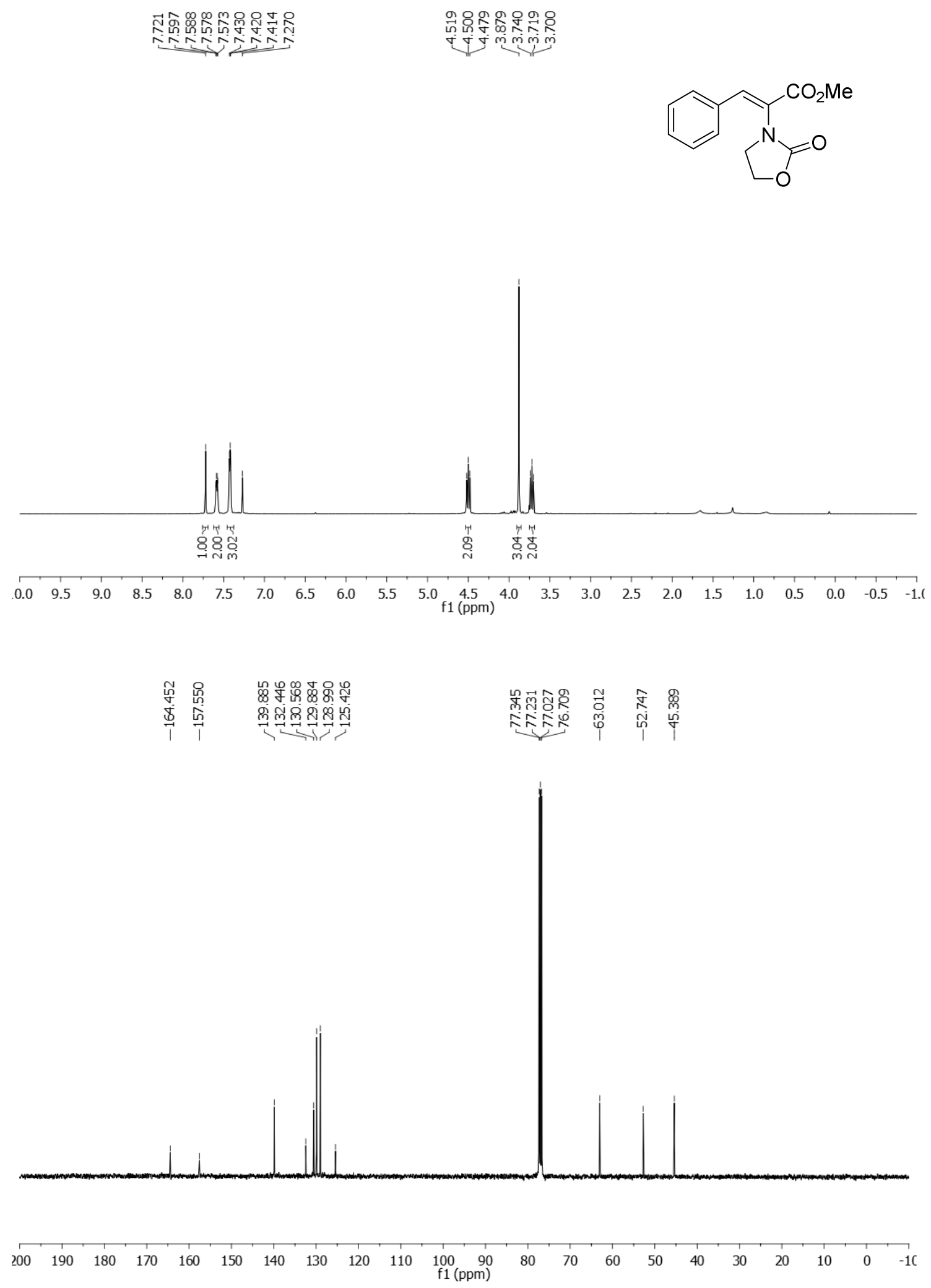


Methyl (Z)-2-(1,3-dioxoisoindolin-2-yl)-3-phenylacrylate (5a)

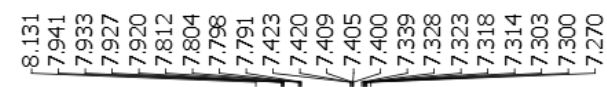

$\stackrel{\substack{\infty \\ \infty}}{\substack{i \\ i}}$<smiles>COC(=O)/C(=C/c1ccccc1)c1ccccc1</smiles>

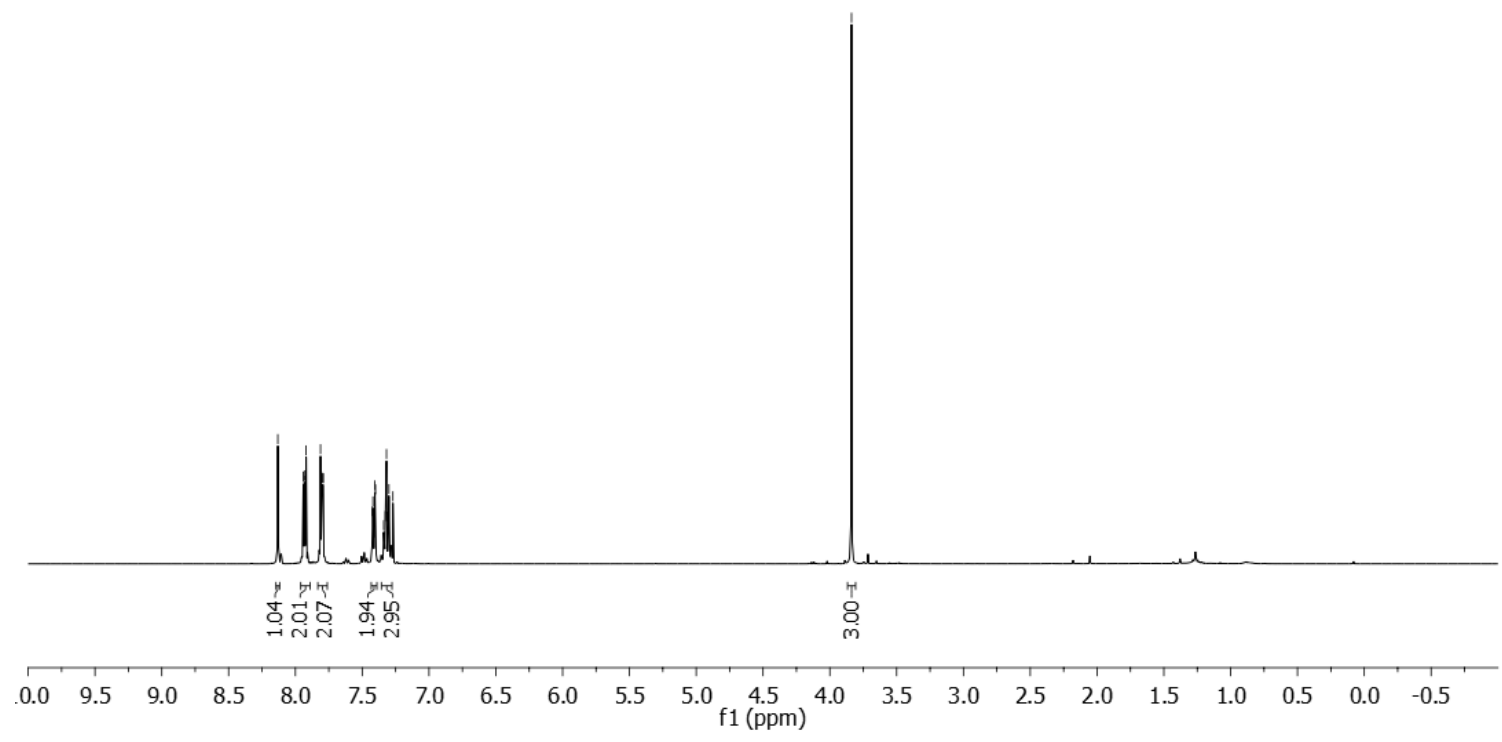

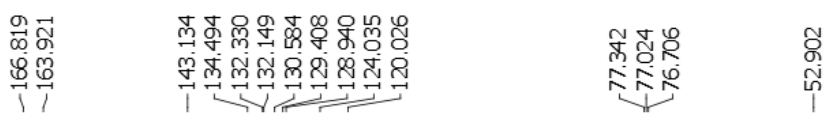

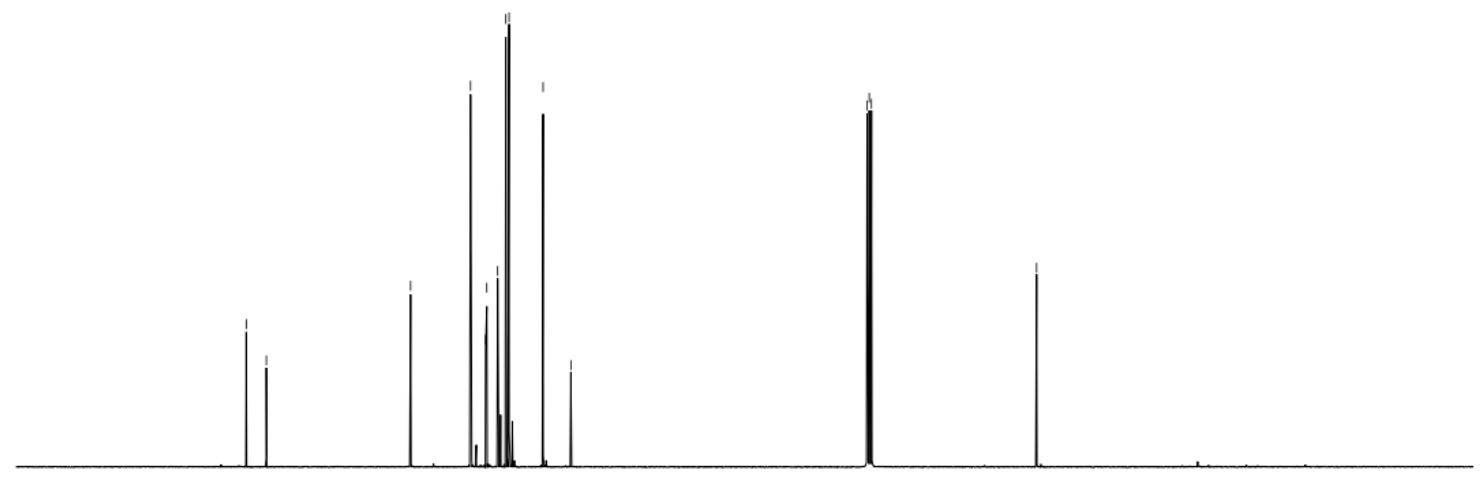

$\begin{array}{lllllllllllllllllllllll}200 & 190 & 180 & 170 & 160 & 150 & 140 & 130 & 120 & 110 & \begin{array}{c}100 \\ \mathrm{f} 1(\mathrm{ppm})\end{array} & 80 & 70 & 60 & 50 & 40 & 30 & 20 & 10 & 0 & -1 \mathrm{C}\end{array}$ 


\section{Methyl (Z)-2-(1,3-dioxoisoindolin-2-yl)-3-(o-/m-/p-tolyl)acrylate (5b)}

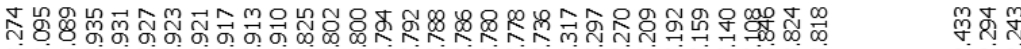

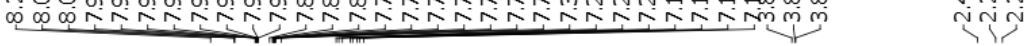<smiles>COC(=O)/C(=C/c1ccccc1)Nc1ccccc1</smiles>
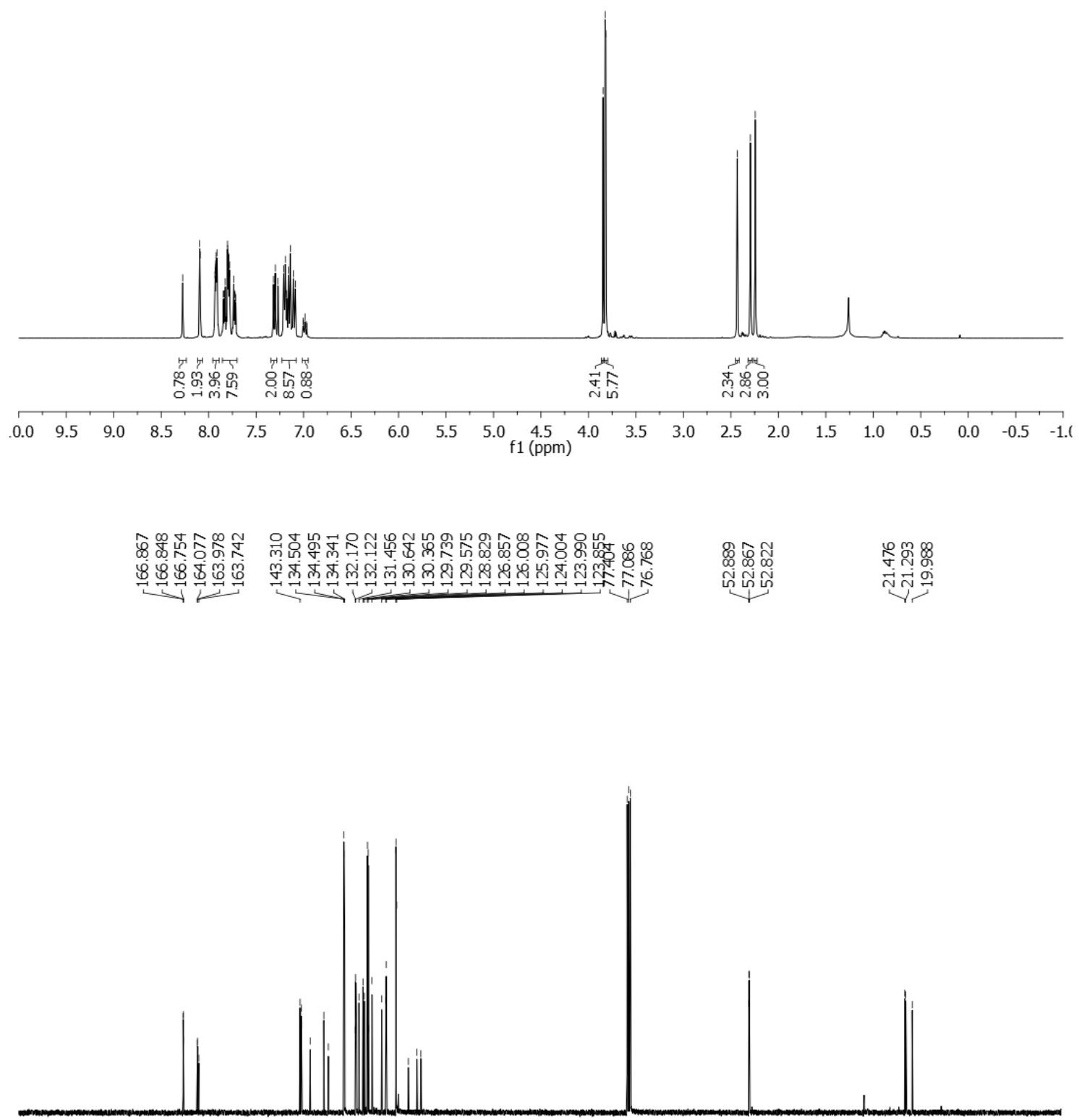

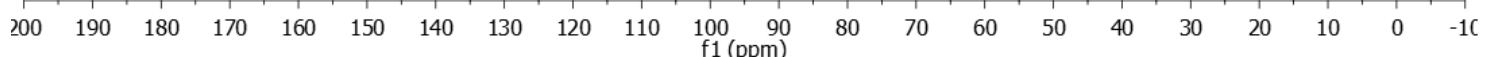


Methyl (Z)-2-(1,3-dioxoisoindolin-2-yl)-3-(2-/3-/4-methoxyphenyl)acrylate (5c)

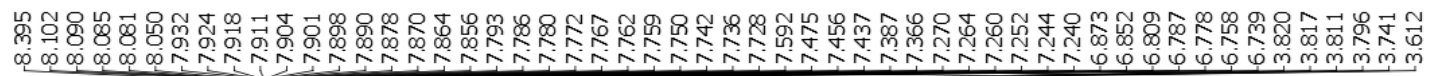

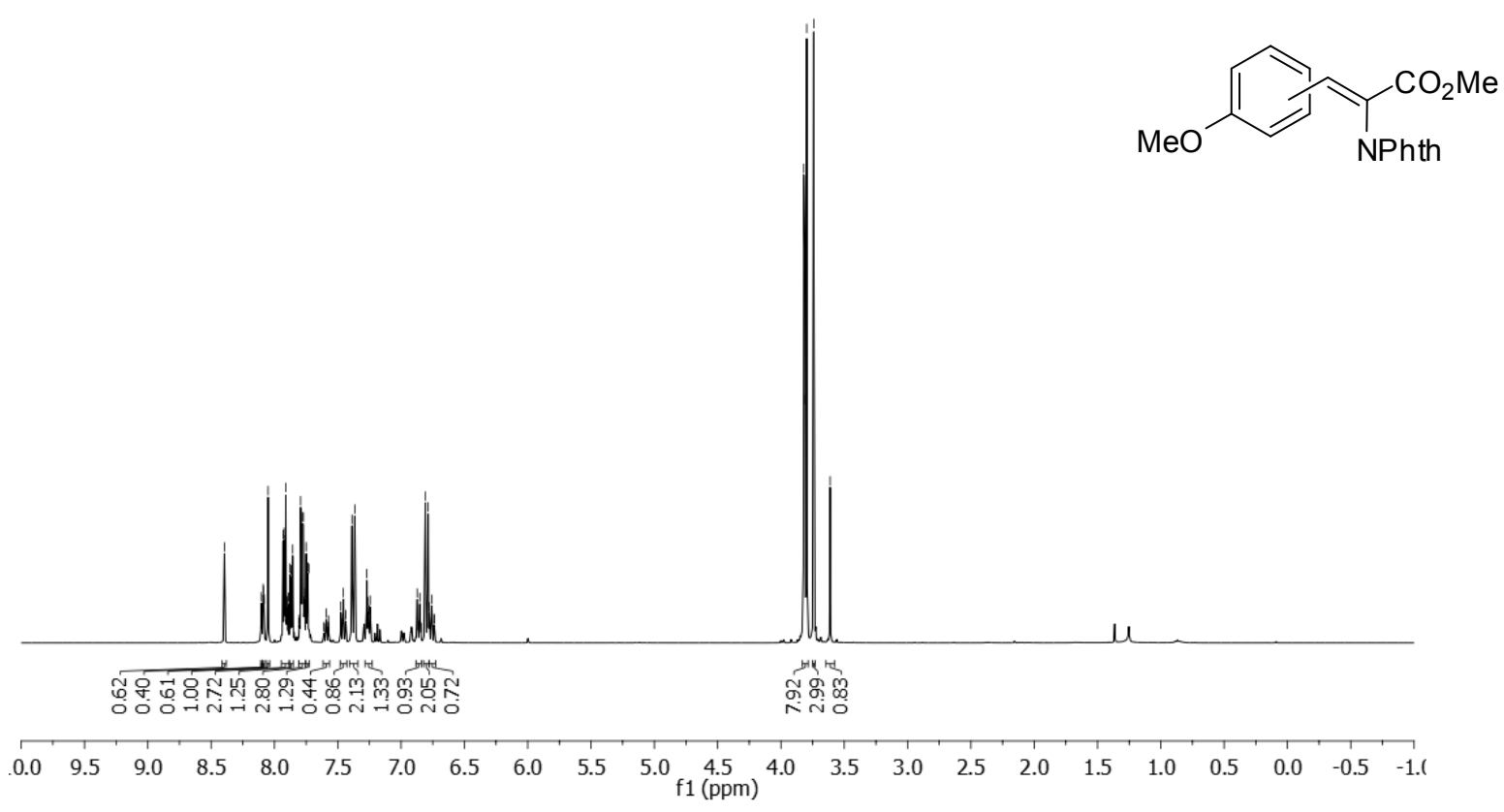

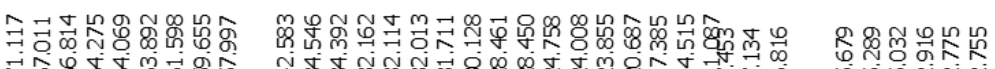

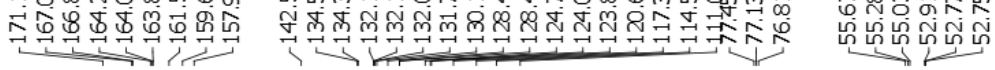

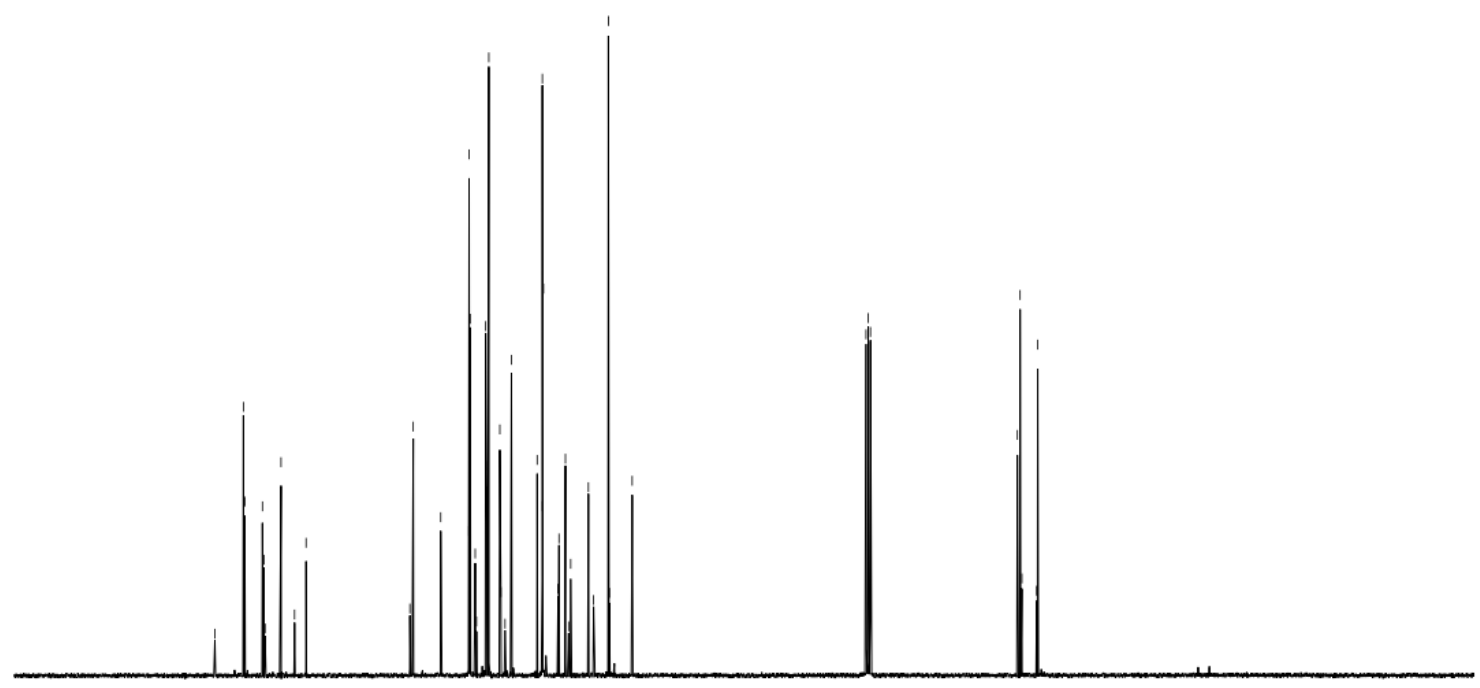

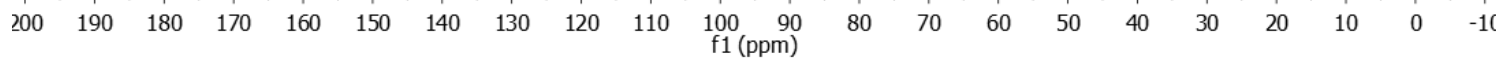


Methyl (Z)-3-(2,5-dimethylphenyl)-2-(1,3-dioxoisoindolin-2-yl)acrylate (5d)

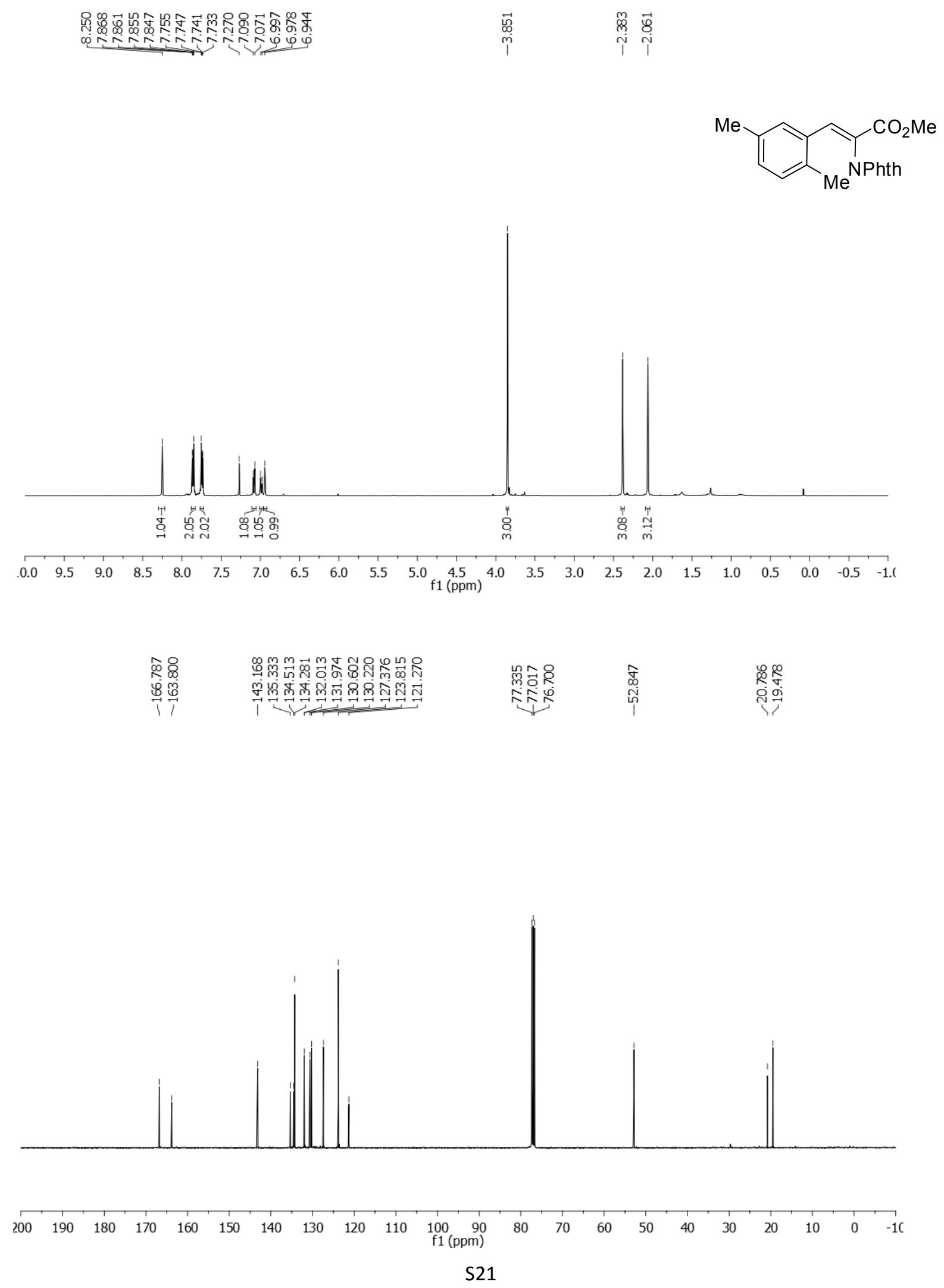


Methyl (Z)-3-(2,5-dimethoxyphenyl)-2-(1,3-dioxoisoindolin-2-yl)acrylate (5e)

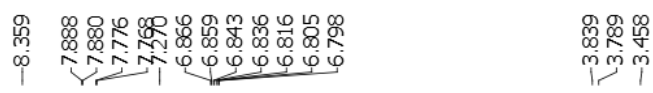<smiles>COC(=O)/C(=C/c1cc(OC)ccc1OC)[NH+](C)c1ccccc1</smiles>

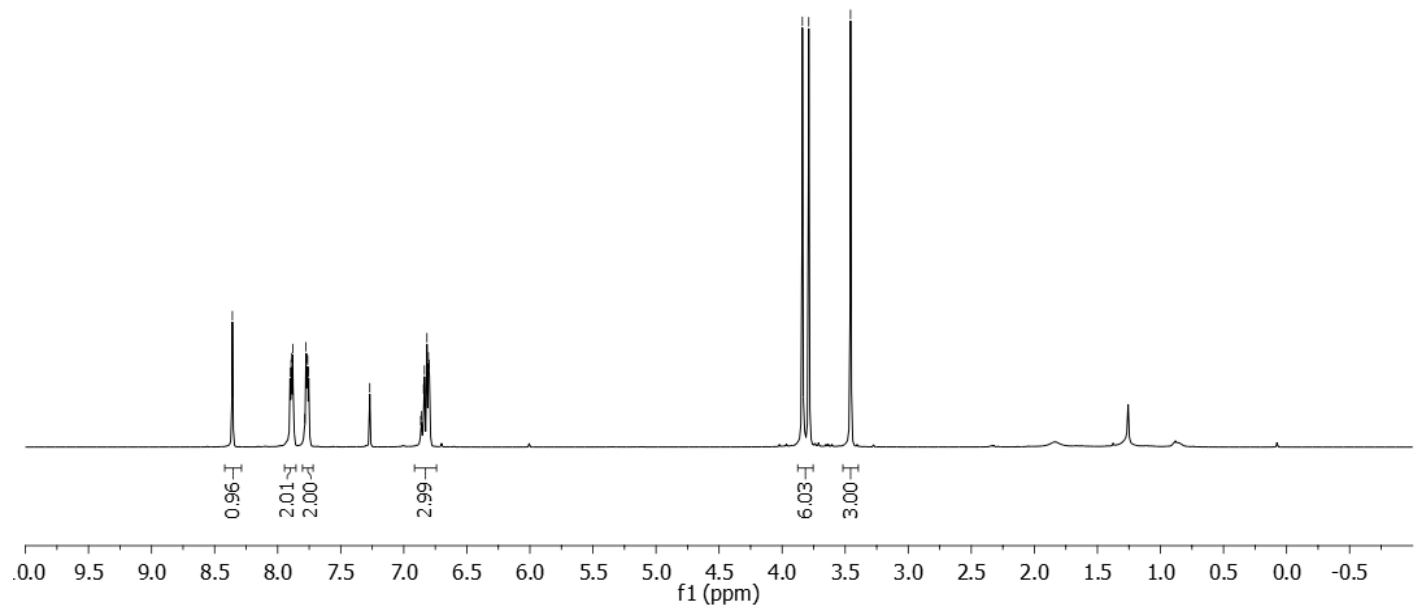

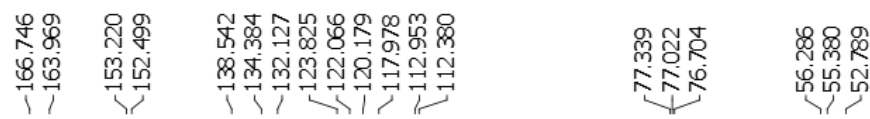

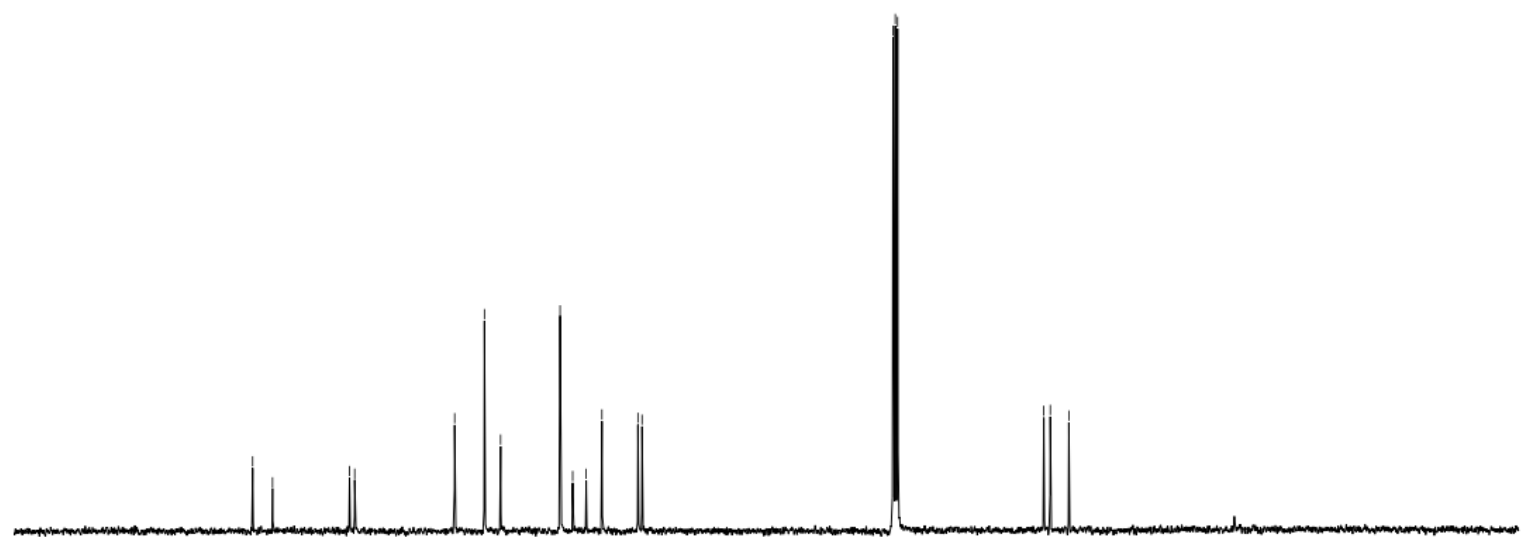

$\begin{array}{lllllllllllllllllllll}200 & 190 & 180 & 170 & 160 & 150 & 140 & 130 & 120 & 110 & \begin{array}{c}100 \\ \mathrm{f} 1(\mathrm{ppm})\end{array} & 80 & 70 & 60 & 50 & 40 & 30 & 20 & 10 & 0 & -1 \mathrm{C}\end{array}$ 
Methyl (Z)-2-(1,3-dioxoisoindolin-2-yl)-3-(2,4,6-trifluorophenyl)acrylate (5f)

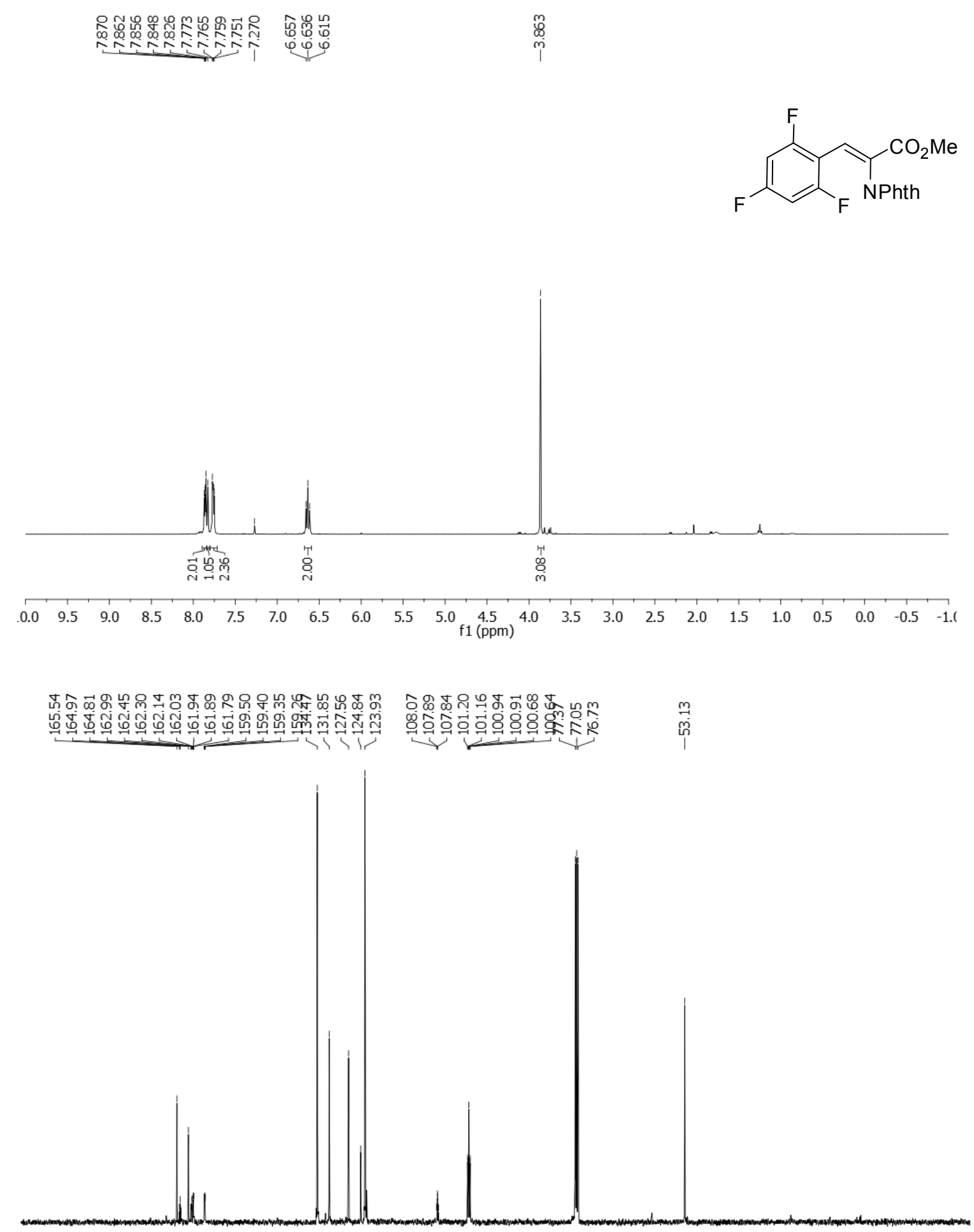

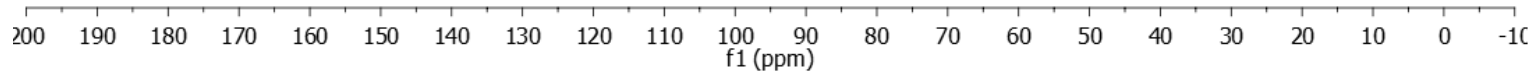




\section{Methyl (Z)-2-(1,3-dioxoisoindolin-2-yl)-3-(perfluorophenyl)acrylate (5g)}

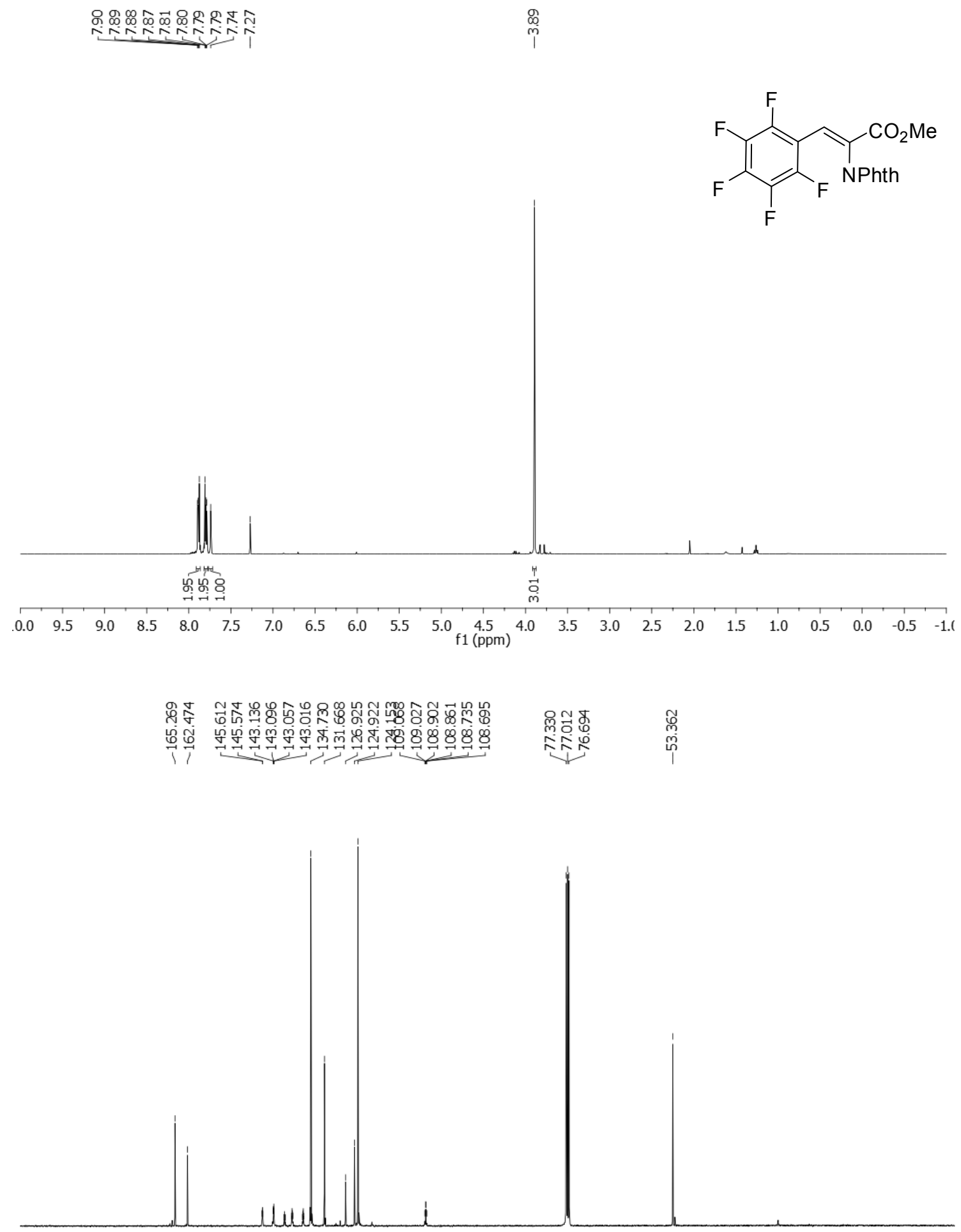

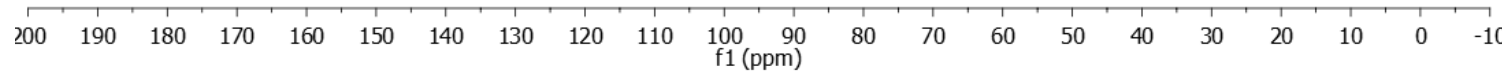




\section{Methyl (Z)-2-(1,3-dioxoisoindolin-2-yl)-3-(naphthalen-1-/2-yl)acrylate (5h)}
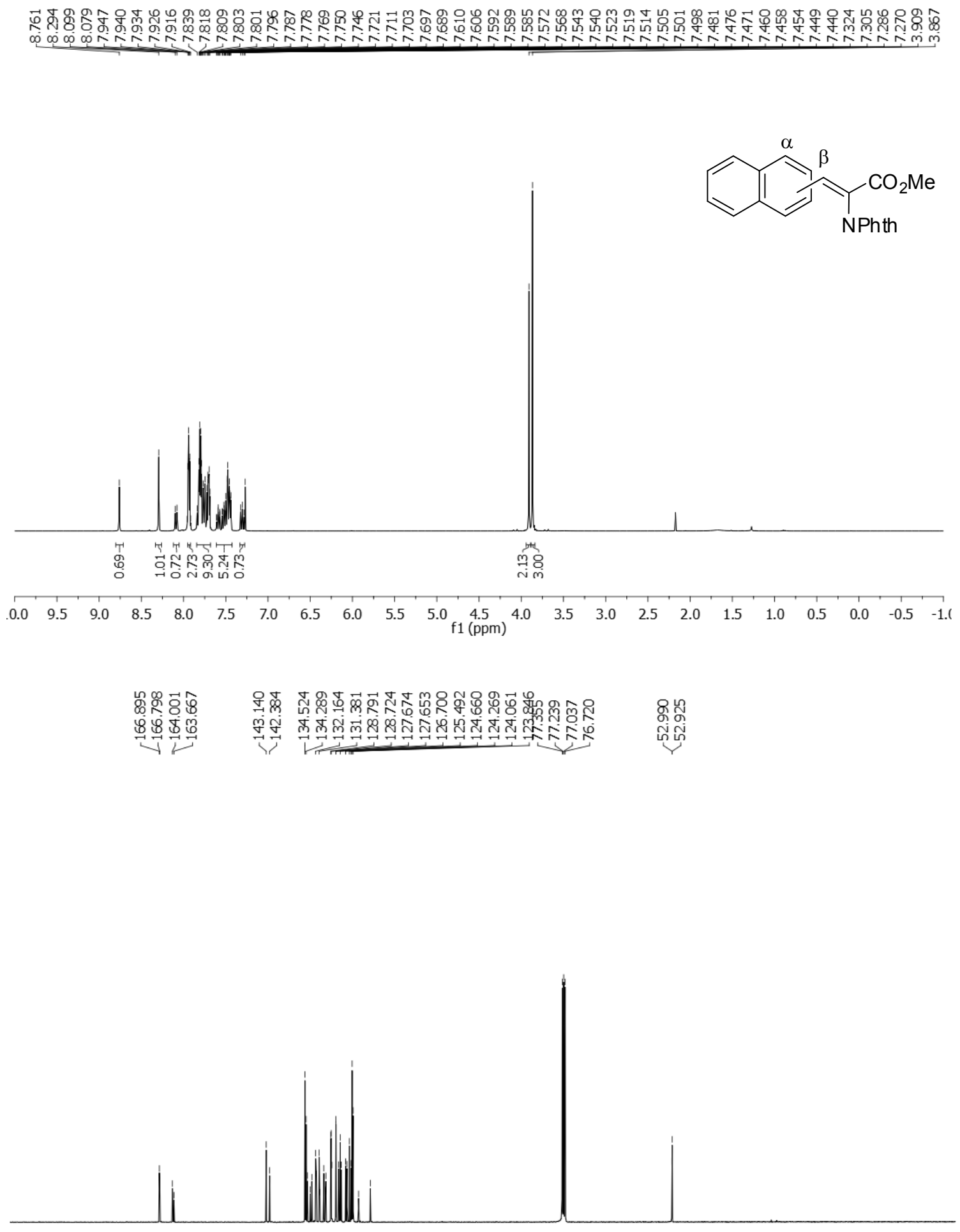

$\begin{array}{llllllllllllllllllllllll}200 & 190 & 180 & 170 & 160 & 150 & 140 & 130 & 120 & 110 & \underset{\mathrm{f} 1(\mathrm{ppm})}{100} & 80 & 70 & 60 & 50 & 40 & 30 & 20 & 10 & 0 & -10\end{array}$ 


\section{Methyl (Z)-3-(2,3-/3,4-dimethylphenyl)-2-(1,3-dioxoisoindolin-2-yl)acrylate (5i)}

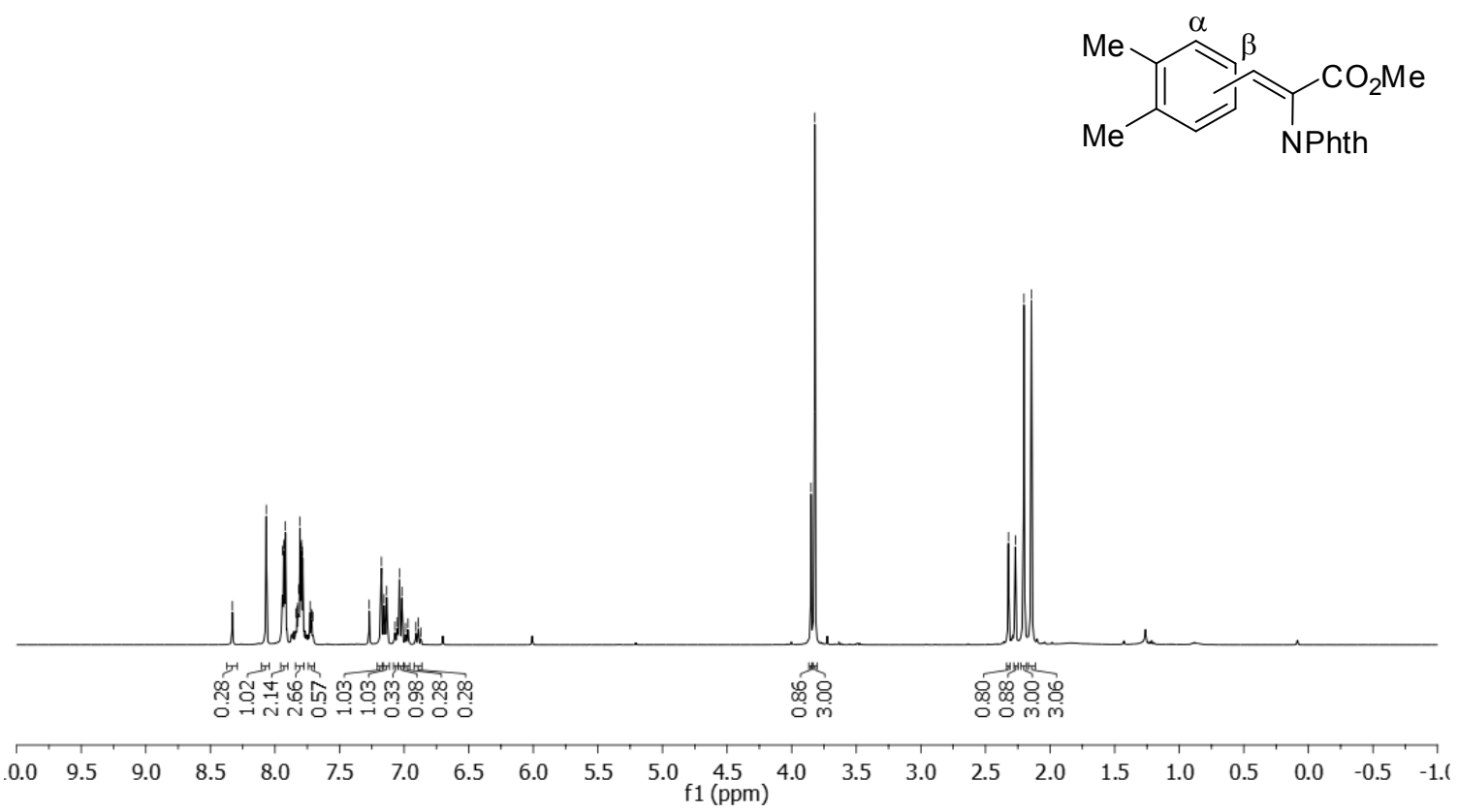

G:/BAF_DMC178_colonna/13/fid
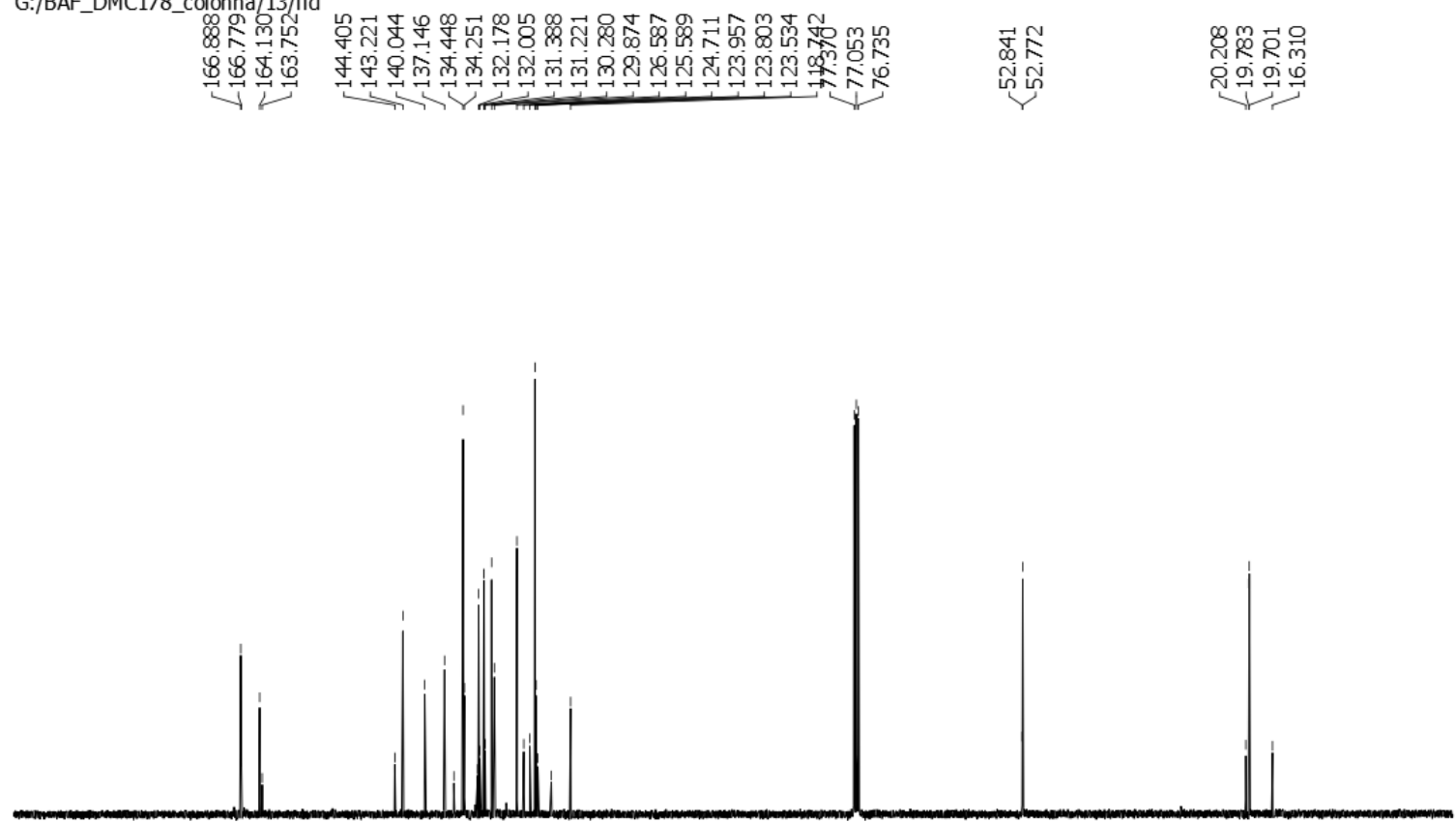

$\begin{array}{lllllllllll}200 & 190 & 180 & 170 & 160 & 150 & 140 & 130 & 120 & 110 & \begin{array}{c}100 \\ \mathrm{f} 1(\mathrm{ppm})\end{array}\end{array}$ 


\section{Methyl (Z)-3-(2,4-/3,5-dimethylphenyl)-2-(1,3-dioxoisoindolin-2-yl)acrylate (5j)}

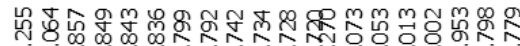

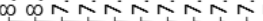

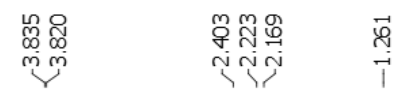

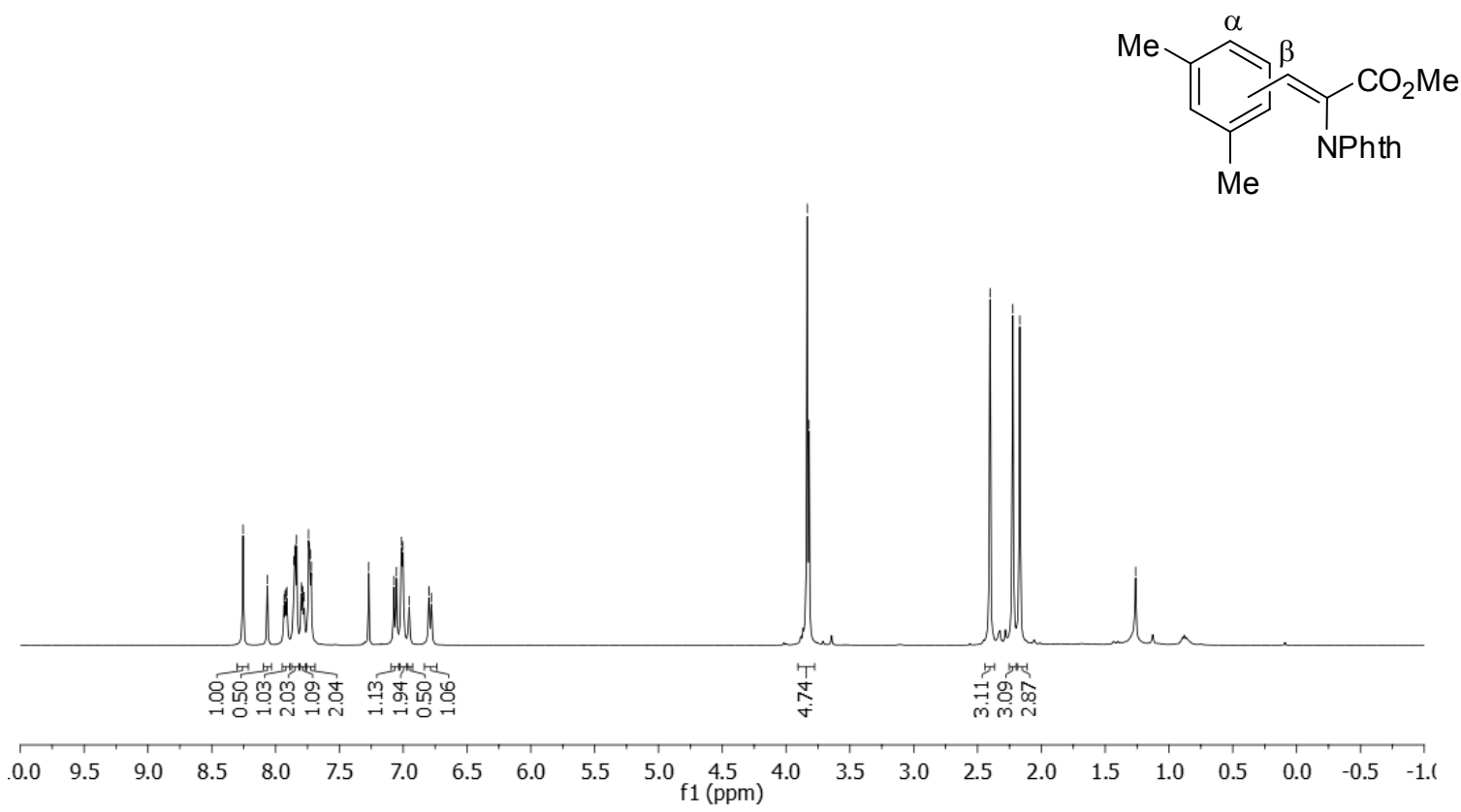

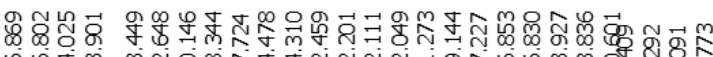

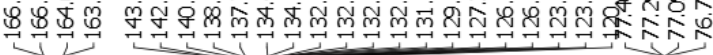

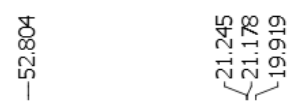

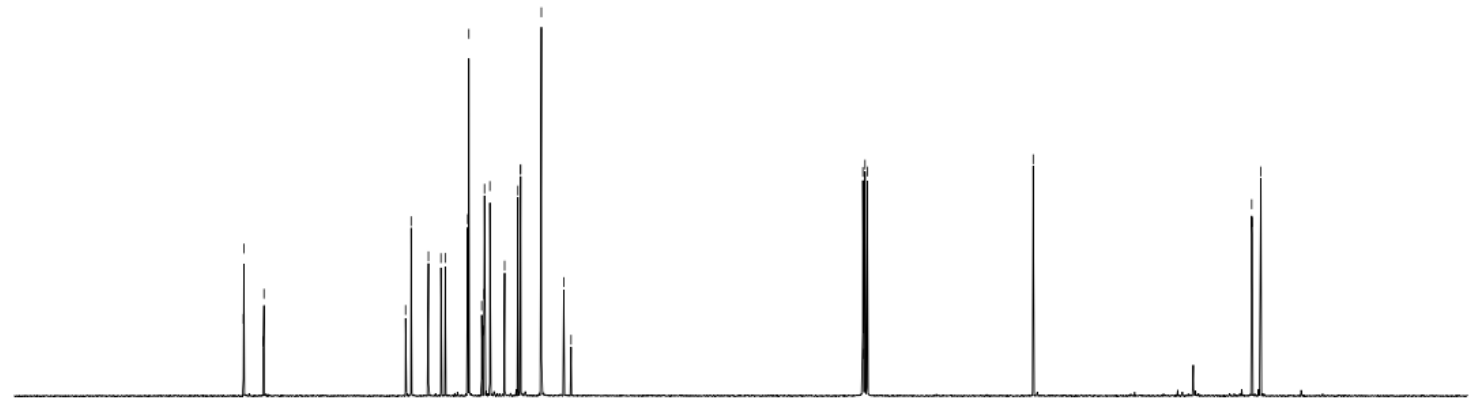


Methyl (Z)-3-(2,3-/3,4-dichlorophenyl)-2-(1,3-dioxoisoindolin-2-yl)acrylate (5k)

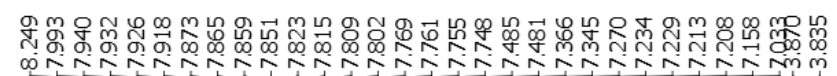

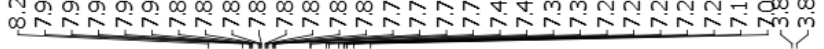

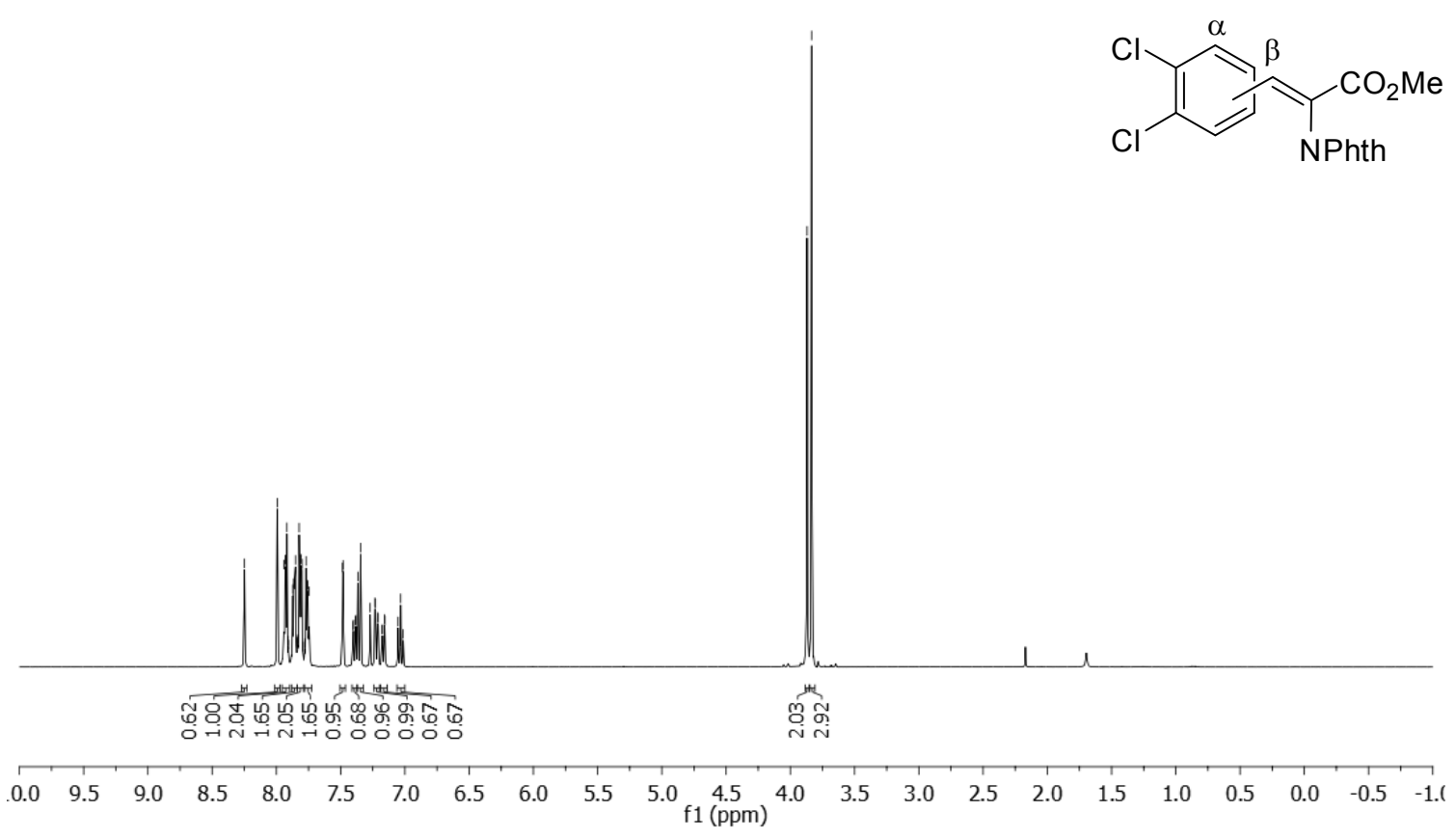

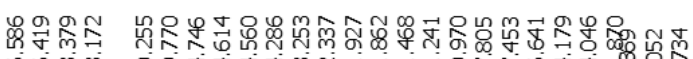

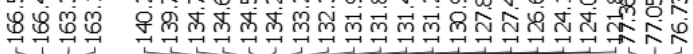

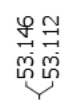

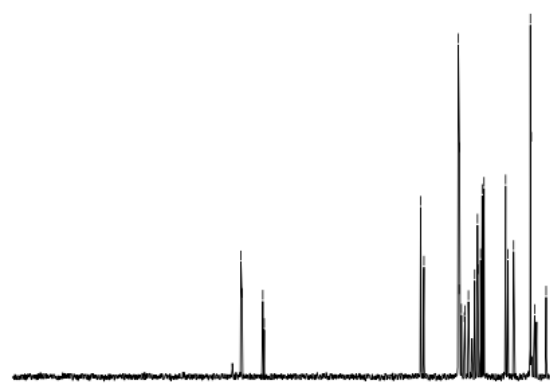

200

$190 \quad 180$

+ ' 17

$150 \quad 140$

$130 \quad 120$

$100 \quad 90$ 


\section{(Z)-Methyl 2-(1,3-dioxoisoindolin-2-yl)-3-(2-/3-/4-(trifluoromethyl)phenyl)acrylate (5l)}

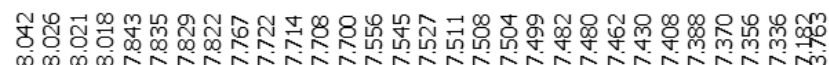

on on<smiles>CC(=O)C(Cc1ccccc1)c1ccc(C(F)(F)F)cc1</smiles>

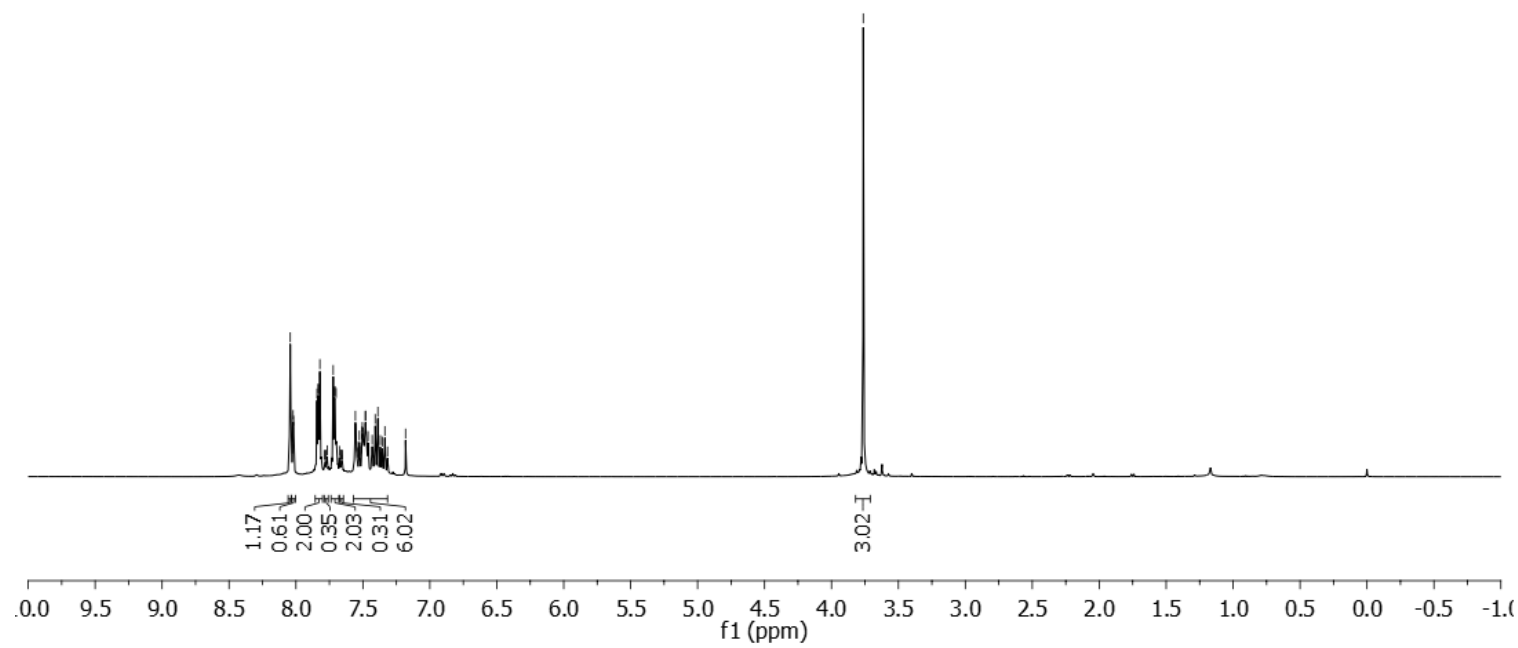

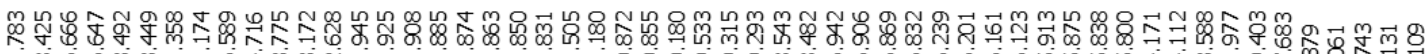

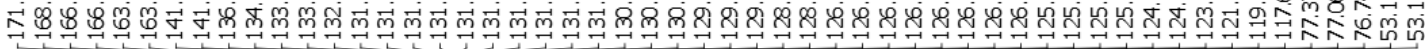

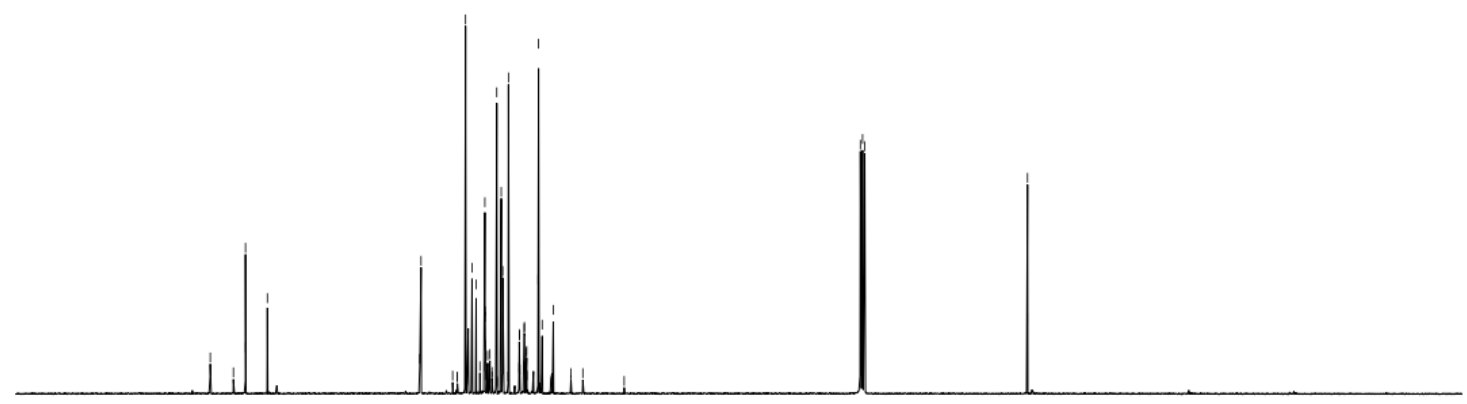

$\begin{array}{lllllllllllllllllllll}200 & 190 & 180 & 170 & 160 & 150 & 140 & 130 & 120 & 110 & \begin{array}{c}100 \\ \mathrm{f} 1(\mathrm{ppm})\end{array} & 80 & 70 & 60 & 50 & 40 & 30 & 20 & 10 & 0 & -1 \mathrm{C}\end{array}$ 


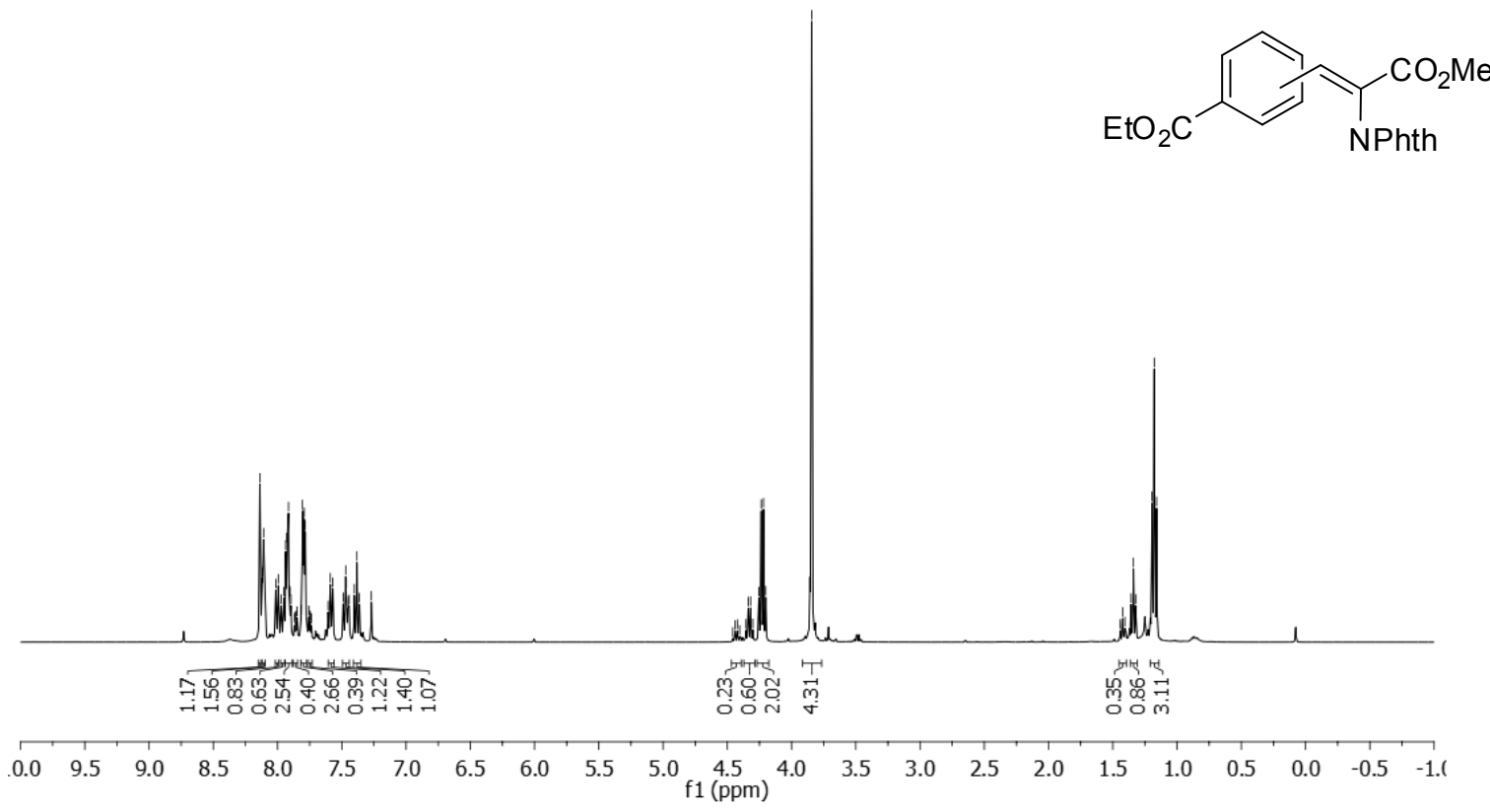

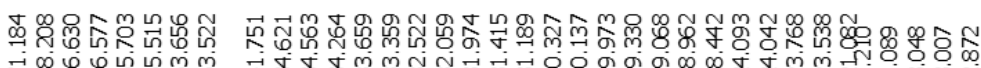

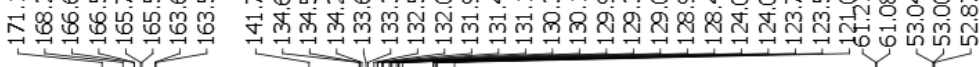

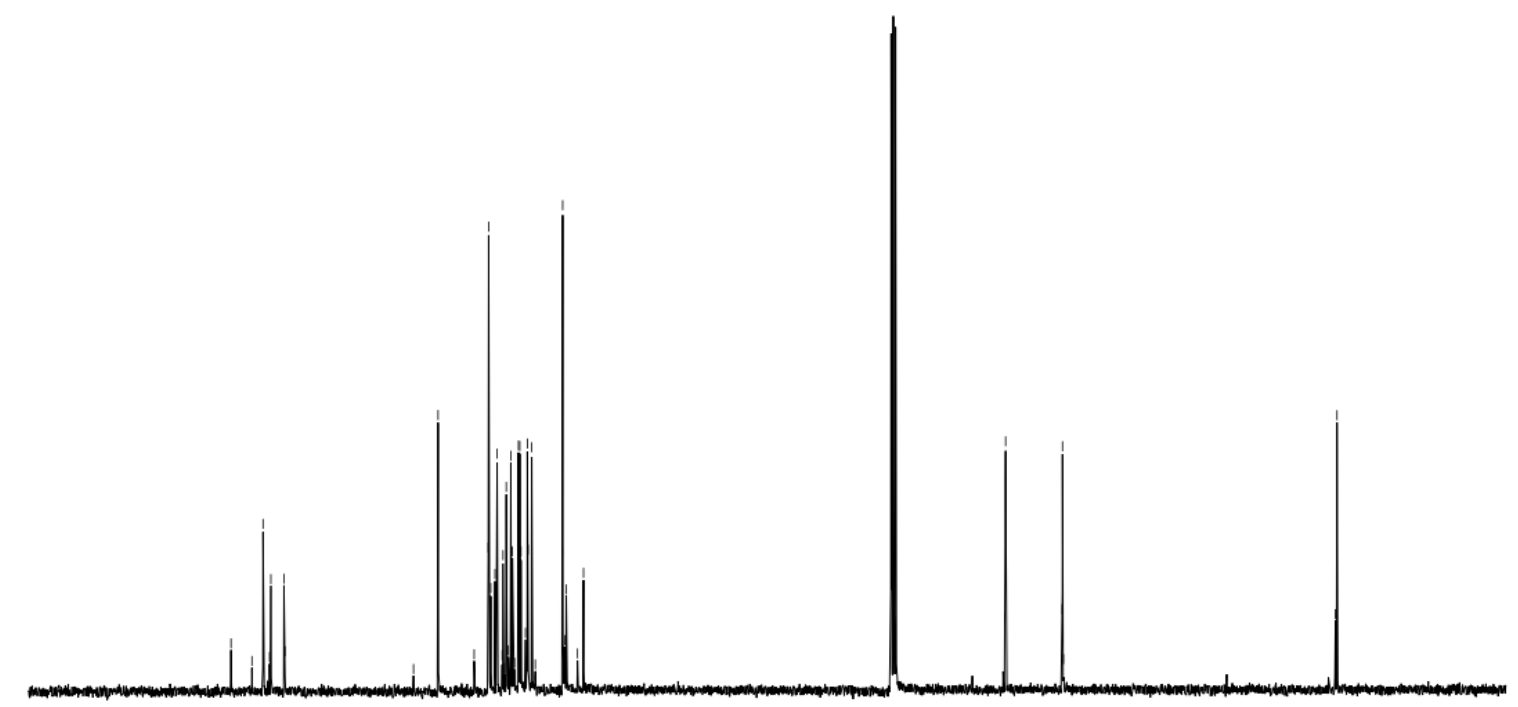

$\begin{array}{llllllllllllllllllllll}200 & 190 & 180 & 170 & 160 & 150 & 140 & 130 & 120 & 110 & \begin{array}{c}100 \\ \mathrm{f} 1(\mathrm{ppm})\end{array} & 80 & 70 & 60 & 50 & 40 & 30 & 20 & 10 & 0 & -10\end{array}$ 
(Z)-Methyl 3-(2-/3-/4-acetylphenyl)-2-(1,3-dioxoisoindolin-2-yl)acrylate (5n)

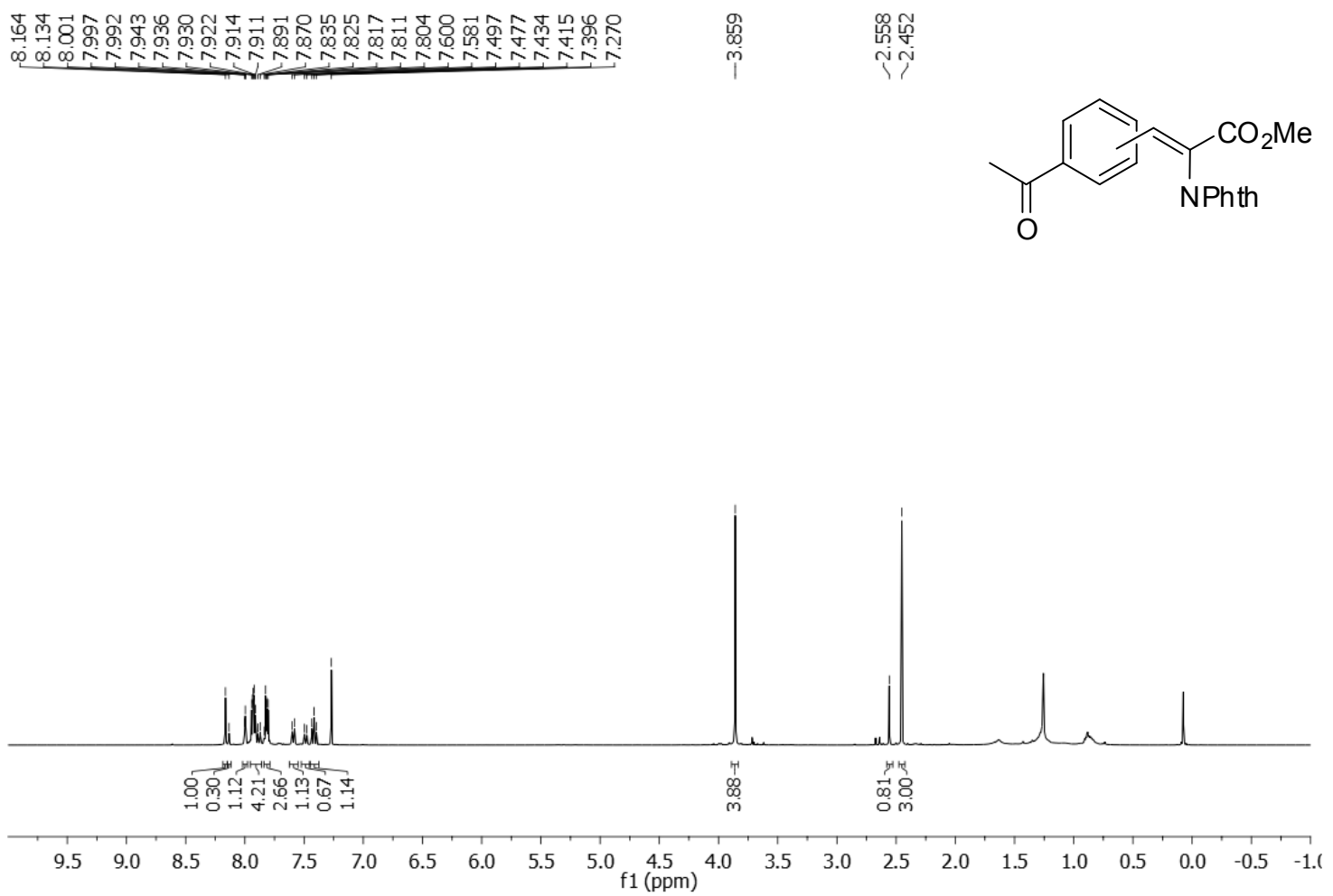

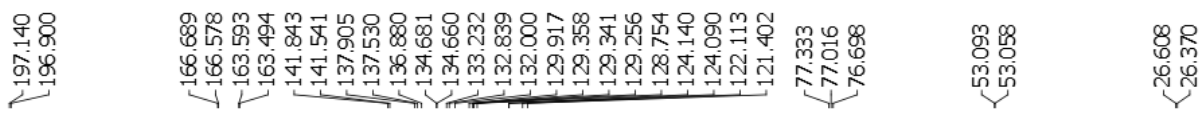

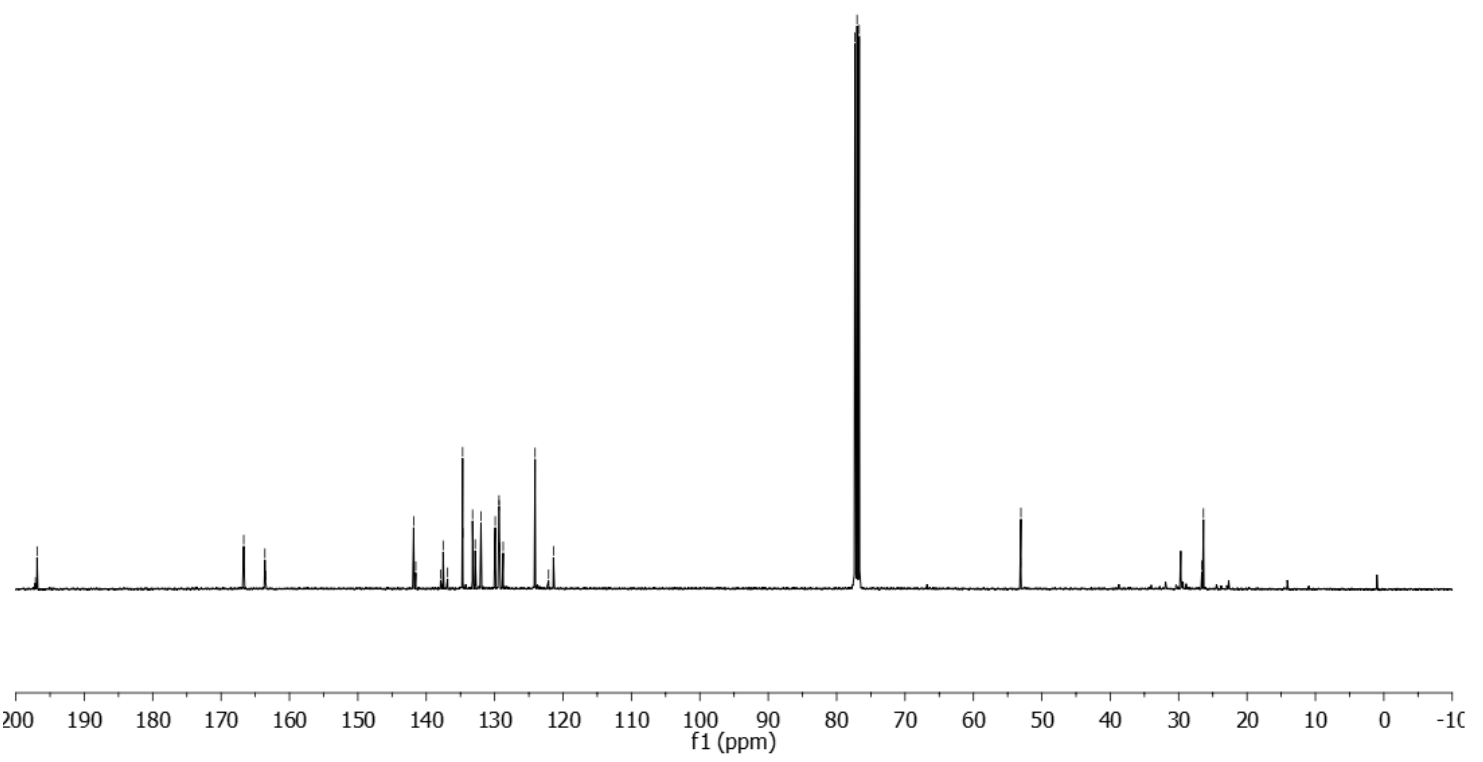


Methyl 2-(1,3-dioxoisoindolin-2-yl)-3-hydroxy-3-(2,4,6-trifluorophenyl)propanoate (7)
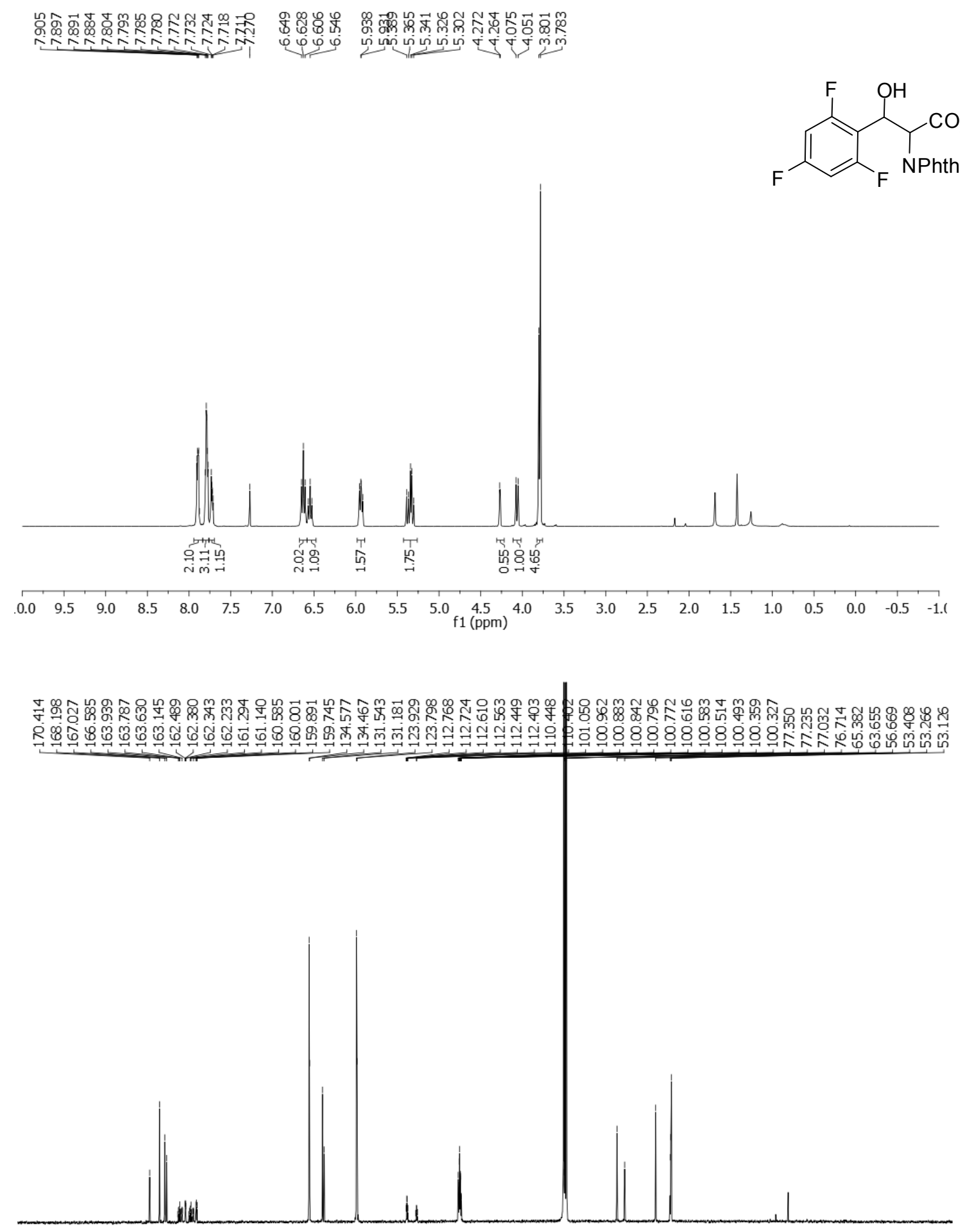

200

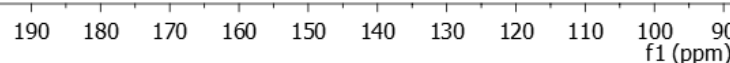

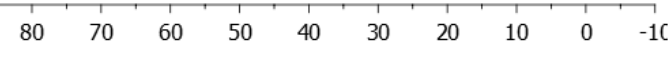




\section{Methyl}

trifluorophenyl)propanoate (8)

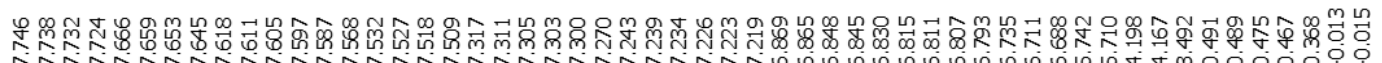

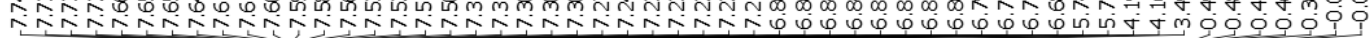

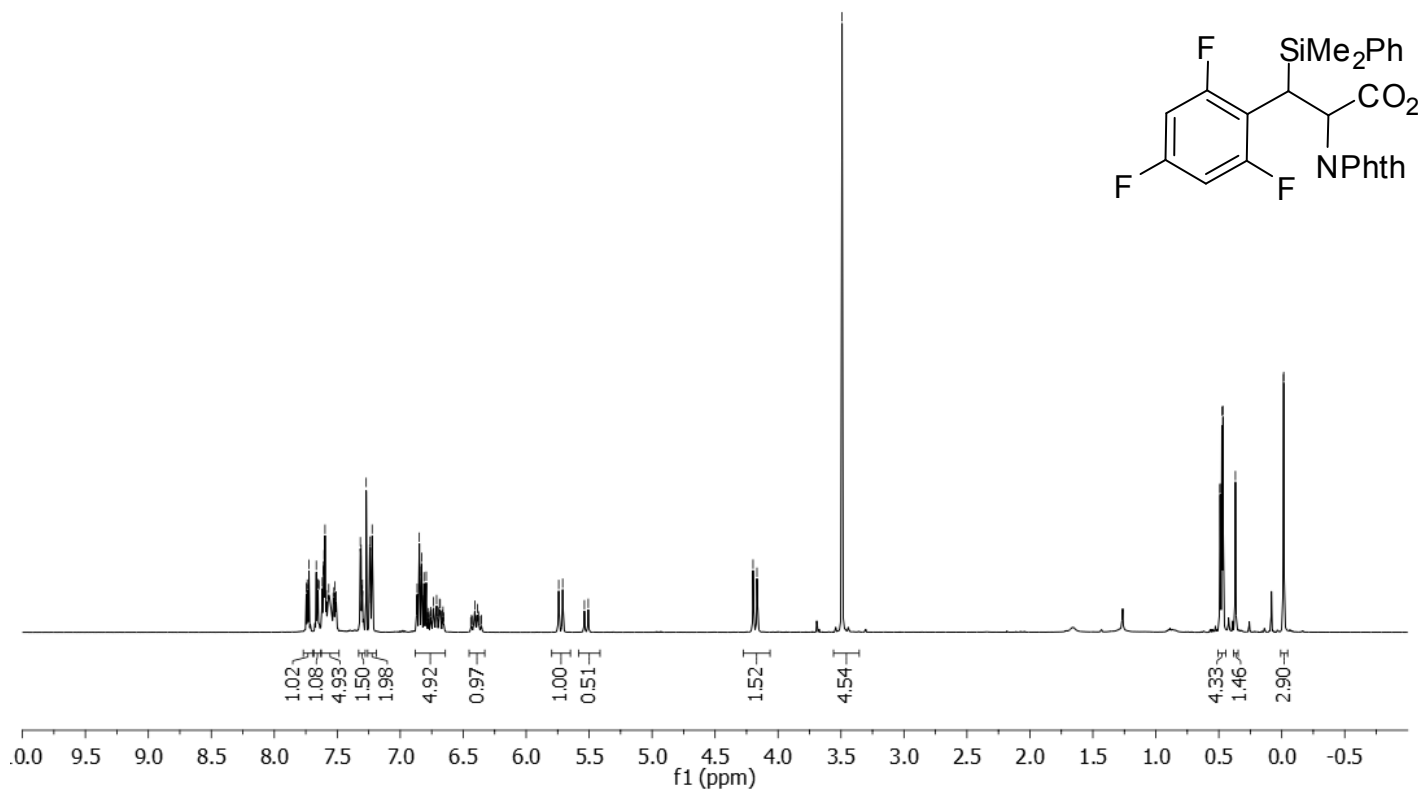

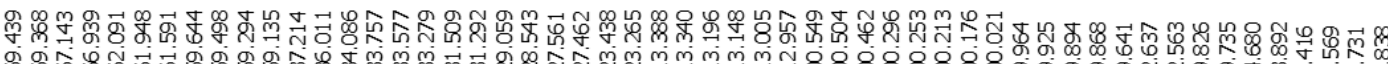

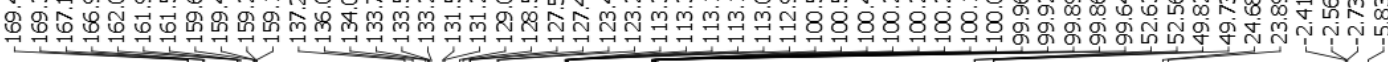

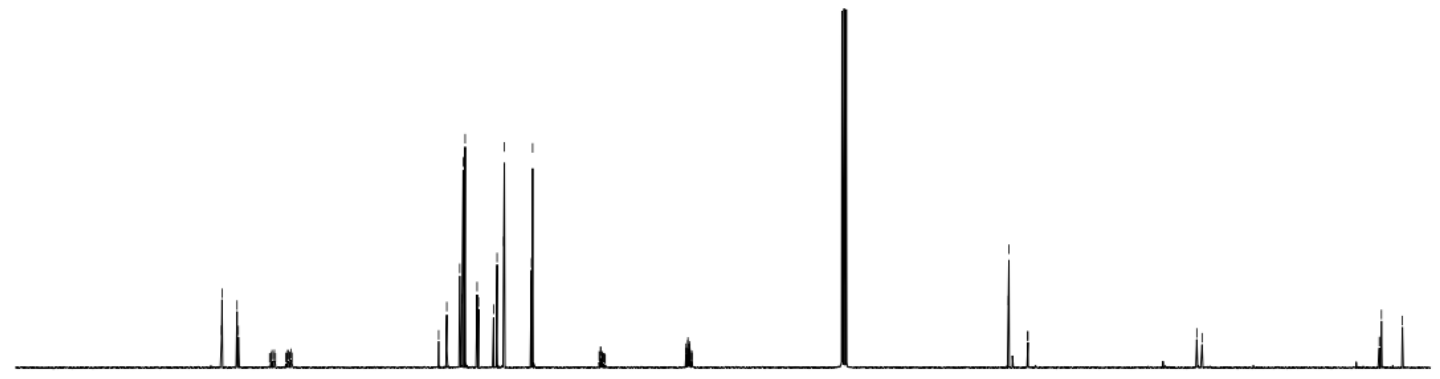

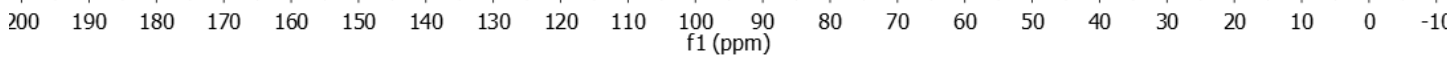


Methyl 2-(1,3-dioxoisoindolin-2-yl)-3-(2,4,6-trifluorophenyl)propanoate (9)

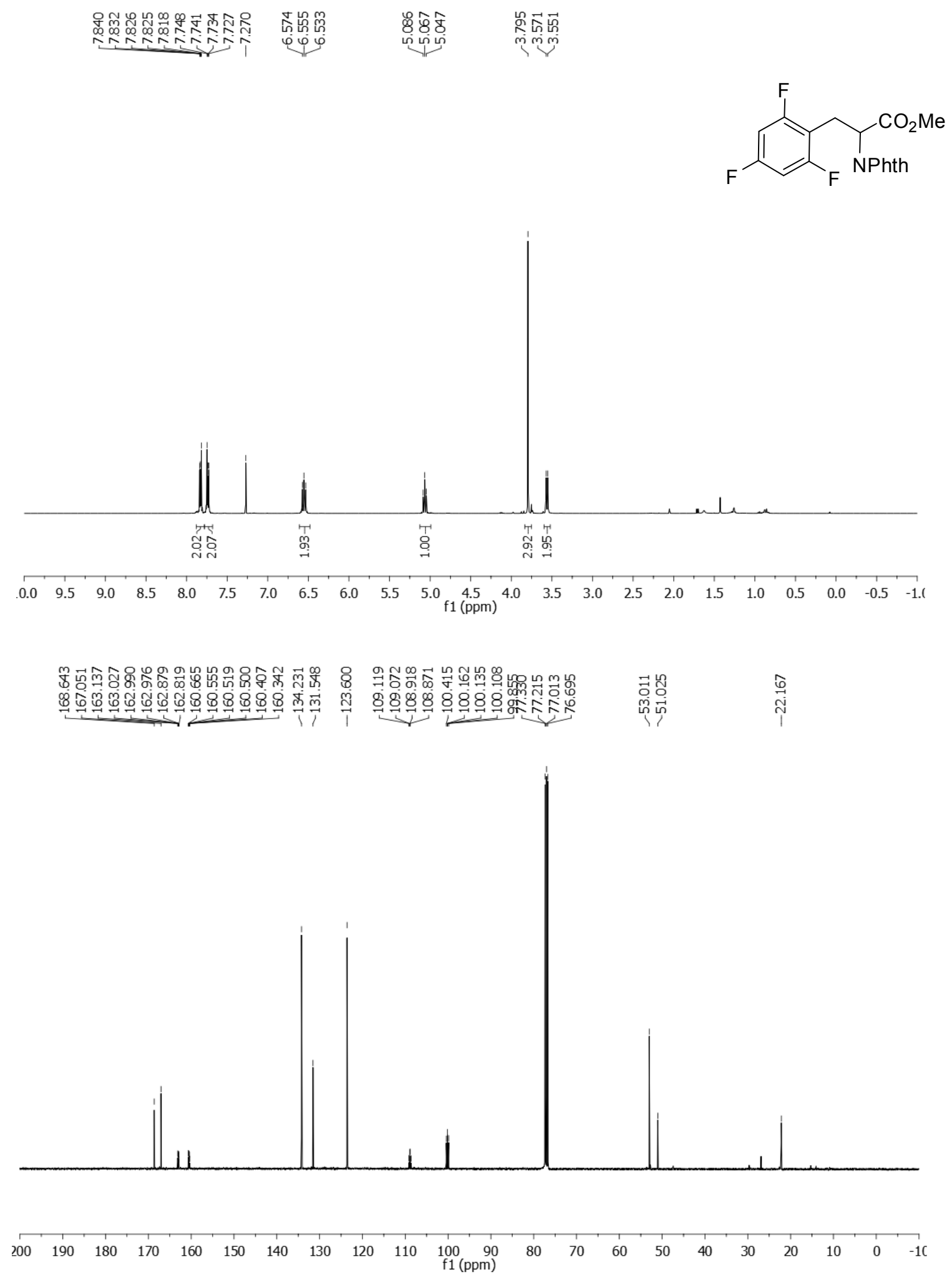


2-((((9H-fluoren-9-yl)methoxy)carbonyl)amino)-3-(2,4,6-trifluorophenyl)propanoic acid (10)

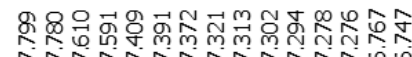

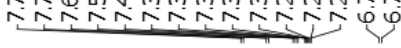

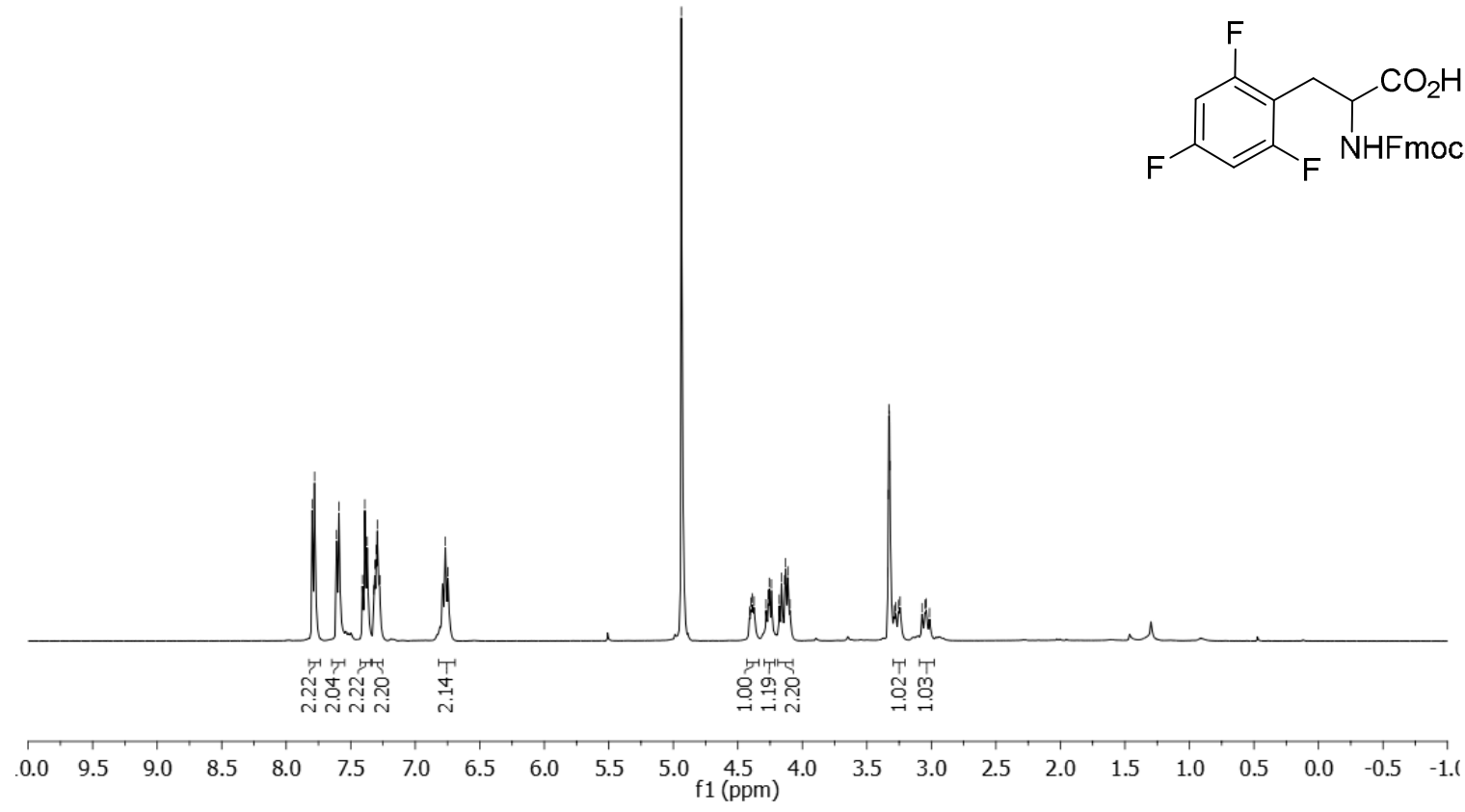

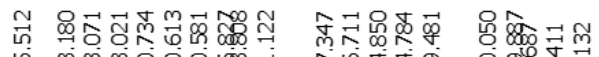

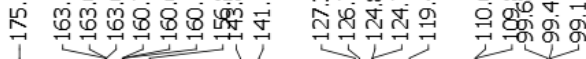
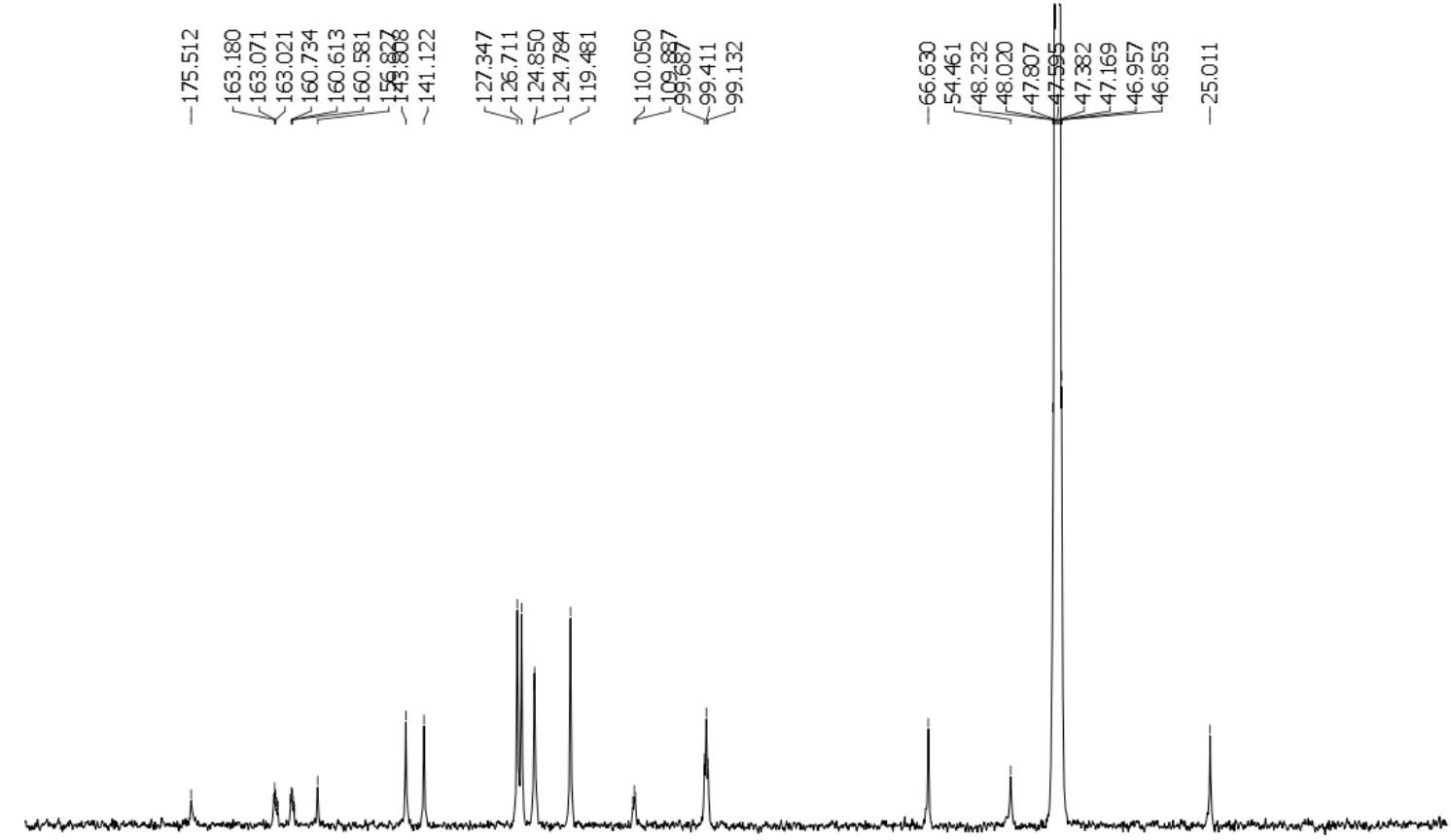

200 